\title{
Dark matter in the standard model?
}

\author{
Christian Gross, ${ }^{1}$ Antonello Polosa, ${ }^{2}$ Alessandro Strumia, ${ }^{1,3}$ Alfredo Urbano, ${ }^{4,3}$ and Wei Xue ${ }^{3}$ \\ ${ }^{1}$ Dipartimento di Fisica dell'Università di Pisa and INFN, Sezione di Pisa, 56127 Pisa, Italy \\ ${ }^{2}$ Dipartimento di Fisica e INFN, Sapienza Università di Roma, I-00185, Roma, Italy \\ ${ }^{3}$ Theoretical Physics Department, CERN, 1211 Geneva 23, Switzerland \\ ${ }^{4}$ INFN, Sezione di Trieste, SISSA, via Bonomea 265, 34136 Trieste, Italy
}

(Received 1 June 2018; published 11 September 2018)

\begin{abstract}
We critically reexamine two possible dark matter candidates within the Standard Model. First, we consider the uuddss hexaquark. Its QCD binding energy could be large enough to make it (quasi)stable. We show that the cosmological dark matter abundance is reproduced thermally if its mass is $1.2 \mathrm{GeV}$. However, we also find that such a mass is excluded by the stability of oxygen nuclei. Second, we consider the possibility that the instability in the Higgs potential leads to the formation of primordial black holes while avoiding vacuum decay during inflation. We show that the nonminimal Higgs coupling to gravity must be as small as allowed by quantum corrections, $\left|\xi_{H}\right|<0.01$. Even so, one must assume that the Universe survived in $e^{120}$ independent regions to fluctuations that lead to vacuum decay with probability $1 / 2$ each.
\end{abstract}

DOI: 10.1103/PhysRevD.98.063005

\section{INTRODUCTION}

In this work we critically reexamine two different intriguing possibilities that challenge the belief that the existence of dark matter (DM) implies new physics beyond the standard model (SM).

\section{A. DM as the uuddss hexaquark}

The binding energy of the hexaquark dibaryon uuddss is expected to be large, given that the presence of the strange quark $s$ allows it to be a scalar, isospin singlet [1], called $H$ or $\mathcal{S}$, and sometimes named sexaquark. A large binding energy might make $\mathcal{S}$ light enough that it is stable or long lived. All possible decay modes of a free $\mathcal{S}$ are kinematically forbidden if $\mathcal{S}$ is lighter than about $1.87 \mathrm{GeV}$. Then $\mathcal{S}$ could be a dark matter candidate within the standard model [2-4].

In Sec. II A we use the recent theoretical and experimental progress about tetra- and pentaquarks to infer the mass of the $\mathcal{S}$ hexaquark. In Sec. II B we present the first cosmological computation of the relic $\mathcal{S}$ abundance, finding that the desired value is reproduced for $M_{\mathcal{S}} \approx 1.2 \mathrm{GeV}$. In Sec. II C we revisit the bound from nuclear stability $(N N \rightarrow \mathcal{S} X$ production within nuclei) at the light of recent

Published by the American Physical Society under the terms of the Creative Commons Attribution 4.0 International license. Further distribution of this work must maintain attribution to the author(s) and the published article's title, journal citation, and DOI. Funded by SCOAP ${ }^{3}$. numerical computations of one key ingredient: the nuclear wave function [5], finding that $\mathcal{S}$ seems excluded.

\section{B. DM as primordial black holes}

Primordial black holes $(\mathrm{PBH})$ are hypothetical relics that can originate from gravitational collapse of sufficiently large density fluctuations. The formation of $\mathrm{PBH}$ is not predicted by standard inflationary cosmology: the primordial inhomogeneities observed on large cosmological scales are too small. PBH can arise in models with large inhomogeneities on small scales, $k \gg \mathrm{Mpc}^{-1}$. PBH as DM candidates are subject to various constraints. $\mathrm{BH}$ lighter than $6 \times 10^{-17} M_{\odot}$ are excluded because of Hawking radiation. $\mathrm{BH}$ heavier than $10^{5} M_{\odot}$ are safely excluded. In the intermediate region, a variety of bounds makes the possibility that $\Omega_{\mathrm{PBH}}=\Omega_{\mathrm{DM}}$ problematic but maybe not excluded - the issue is presently subject to an intense debate. According to [6] DM as PBH with mass $M \sim$ $10^{-15} M_{\odot}$ is not excluded, as previously believed. And the HSC/Subaru microlensing constraint on $\mathrm{PBH}$ [7] is partially in the wave optics region. This can invalidate its bound below $\sim 10^{-11} M_{\odot}$.

Many ad hoc models that can produce PBH as DM have been proposed. Recently the authors of [8] claimed that a mechanism of this type is present within the Standard Model given that, for present best-fit values of the measured SM parameters, the SM Higgs potential is unstable at $h>h_{\max } \sim 10^{10} \mathrm{GeV}$ [9]. We here critically reexamine the viability of the proposed mechanism, which assumes that the Higgs, at some point during inflation, has a 
homogeneous vacuum expectation value mildly above the top of the barrier and starts rolling down. When inflation ends, reheating adds a large thermal mass to the effective Higgs potential, which, under certain conditions, brings the Higgs back to the origin, $h=0$ [10]. If falling stops very close to the disaster, this process generates inhomogeneities that lead to the formation of primordial black holes. In Sec. III we extend the computations of [8] adding a nonvanishing nonminimal coupling $\xi_{H}$ of the Higgs to gravity, which is unavoidably generated by quantum effects [11]. We find that $\xi_{H}$ must be as small as allowed by quantum effects. Under the assumptions made in [8] we reproduce their results; however, in Sec. III F we also find that such assumptions imply an extreme fine-tuning.

The first mechanism is affected by the observed baryon asymmetry, but does not depend on the unknown physics that generates the baryon asymmetry. The second possibility depends on inflation, but the mechanism only depends on the (unknown) value of the Hubble constant during inflation. In both cases the DM candidate is part of the SM. Conclusions are given in Sec. IV.

\section{DM AS THE uuddss HEXAQUARK}

The hexaquark $\mathcal{S}=$ uuddss is stable if all its possible decay modes are kinematically closed:

$$
\mathcal{S} \rightarrow \begin{cases}\mathrm{d} e \bar{\nu}_{e} & M_{\mathcal{S}}<M_{\mathrm{d}}+M_{e}=1.8761 \mathrm{GeV}, \\ \operatorname{ppee}_{e} \bar{\nu}_{e} & M_{\mathcal{S}}<2\left(M_{p}+M_{e}\right)=1.8775 \mathrm{GeV}, \\ p e \bar{\nu}_{e} n & M_{\mathcal{S}}<M_{p}+M_{e}+M_{n}=1.8783 \mathrm{GeV} \\ n n & M_{\mathcal{S}}<2 M_{n}=1.8791 \mathrm{GeV} .\end{cases}
$$

A stable $\mathcal{S}$ is a possible DM candidate. A too light $\mathcal{S}$ can make nuclei unstable. Scanning over all stable nuclei, we find that none of them gets destabilized by single $\mathcal{S}$ emission if $M_{\mathcal{S}}>1.874 \mathrm{GeV}$, with ${ }^{6} \mathrm{Li}$ giving the potentially highest sensitivity to $M_{\mathcal{S}}$.

\section{A. Mass of the hexaquark from a diquark model}

We estimate the mass of the hexaquark $\mathcal{S}$ viewing it as a neutral scalar dibaryon constituted by three spin zero diquarks

$$
\mathcal{S}=\epsilon^{\alpha \beta \gamma}[u d]_{\alpha, s=0}[u s]_{\beta, s=0}[d s]_{\gamma, s=0},
$$

where $\alpha, \beta, \gamma$ are color indices. This is possible thanks to the strange $s$ quark, while spin zero diquarks of the kind $[u u],[d d],[s s]$ are forbidden by Fermi statistics because of antisymmetry in color and spin. We assume the effective Hamiltonian for the hexaquark [12]

$$
H=\sum_{i \neq j=\{u, d, s\}}\left(m_{i j}+2 \kappa_{i j} \boldsymbol{S}_{i} \cdot \boldsymbol{S}_{j}\right),
$$

where the $\kappa_{i j}$ are effective couplings determined by the strong interactions at low energies, color factors, quark masses, and wave functions at the origin. The $m_{i j}$ are the masses of the diquarks in $\mathcal{S}$ made of $i$ and $j$ constituent quarks [13]. $S_{i}$ is the spin of the $i$ th quark. Another important assumption, which is well motivated by studies on tetraquarks [12], is that spin-spin interactions are essentially within diquarks and zero outside, as if they were sufficiently separated in space.

Considering diquark masses to be additive in the constituent quark masses, and taking $q$ and $s$ constituent quark masses from the baryons, one finds

$$
m_{[q q]} \simeq 0.72 \mathrm{GeV}, \quad m_{[q s]} \sim 0.90 \mathrm{GeV}, \quad q=\{u, d\} .
$$

The chromomagnetic couplings $\kappa_{i j}$ could be derived as well in the constituent quark model using data on baryons

$$
\kappa_{q q} \simeq 0.10 \mathrm{GeV}, \quad \kappa_{q s} \simeq 0.06 \mathrm{GeV} .
$$

However, it is known that to reproduce the masses of light scalar mesons, interpreted as tetraquarks, $\sigma(500), f_{0}(980)$, $a_{0}(980), \kappa[14]$, we need

$$
\kappa_{q q} \simeq 0.33 \mathrm{GeV}, \quad \kappa_{q s} \simeq 0.27 \mathrm{GeV} .
$$

Spin-spin couplings in tetraquarks are found to be about a factor of 4 larger compared to the spin-spin couplings among the same pairs of quarks in the baryons, which also make diquarks. It is difficult to assess if this would change within a hexaquark. At any rate we can attempt a simple mass formula for $\mathcal{S}$,

$$
M_{\mathcal{S}}=\left(m_{[q q]}-3 / 2 \kappa_{q q}\right)+2\left(m_{[q s]}-3 / 2 \kappa_{q s}\right),
$$

which in terms of light tetraquark masses means $M_{\mathcal{S}}=M_{\sigma} / 2+M_{f_{0}}$. Using the determination of chromomagnetic couplings from baryons we would obtain

$$
M_{\mathcal{S}} \approx 2.17 \mathrm{GeV}
$$

whereas keeping the chromomagnetic couplings needed to fit light tetraquarks gives

$$
M_{\mathcal{S}} \approx 1.2 \mathrm{GeV}
$$

if the same values for the chromomagnetic couplings to fit light tetraquark masses are taken [or $1.4 \mathrm{GeV}$ using (6)]. There is quite a lot of experimental information on tetraquarks [12], whereas hexaquarks, for the moment, are purely hypothetical objects. On purely qualitative grounds we might expect that the mass of $\mathcal{S}$ could be closer to the heavier value being a dibaryon and not a dimeson (tetraquark) like light scalar mesons. 

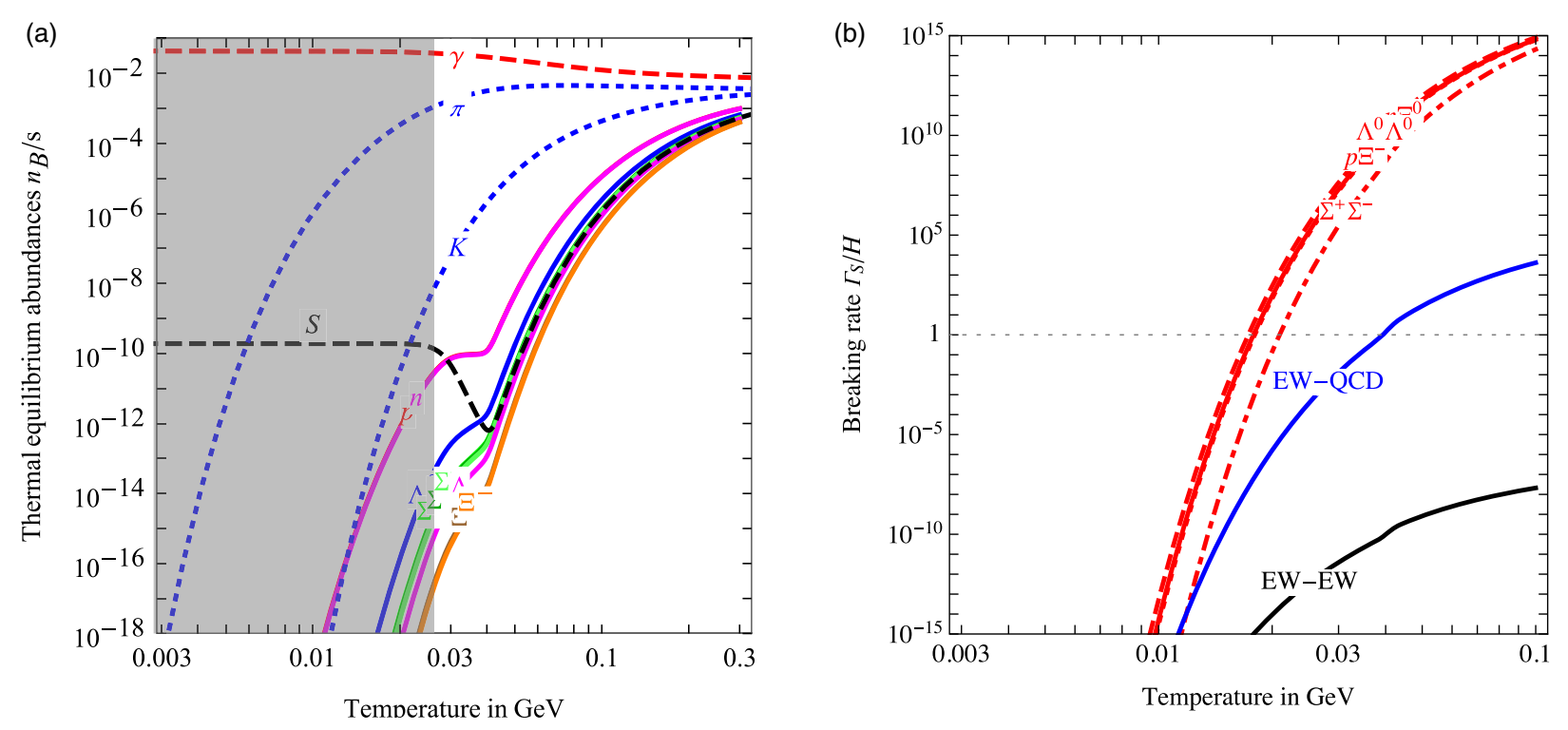

FIG. 1. Left: Thermal equilibrium values of hadron abundances assuming $Y_{B \mathcal{S}}=7.6 \times 10^{-10}$ and $M_{\mathcal{S}}=1.2 \mathrm{GeV}$ : the desired abundances are obtained if decoupling of $\mathcal{S}$ interactions occurs at $T \sim 25 \mathrm{MeV}$. We see that in this phase antiparticles are negligible. Right: Thermal equilibrium values of $\Gamma_{\mathcal{S}} / H$.

In the absence of any other experimental information it is impossible to provide an estimate of the theoretical uncertainty on $M_{\mathcal{S}}$.

Lattice computations performed at unphysical values of quark masses find small values for the $\mathcal{S}$ binding energy, about 13, 75, $20 \mathrm{MeV}$ [15-17]. Extrapolations to physical quark masses suggest that $\mathcal{S}$ does not have a sizable binding energy; see, e.g., [18]. Furthermore, the binding energy of the deuteron is small, indirectly disfavoring a very large binding energy for the (somehow similar) $\mathcal{S}$, which might too be a moleculelike state. ${ }^{1}$ Despite this, we overoptimistically treat $M_{\mathcal{S}}$ as a free parameter in the following.

We also notice that the $\mathcal{S}$ particle could be much larger than what is envisaged in [2-4] and that its coupling to photons, in the case of 2-3 fm size (see the considerations on diquark-diquark repulsion at small distances in [19]), could be relevant for momentum transfers $k$ as small as $k \sim 60 \mathrm{MeV}$, compared to $k \approx 0.5 \mathrm{GeV}$ considered by Farrar.

\section{B. Cosmological relic density of the hexaquark}

We here compute the relic density of $\mathcal{S}$ dark matter, studying if it can match the measured value $\Omega_{\mathrm{DM}} h^{2}=$ 0.1186 , i.e., $\Omega_{\mathrm{DM}} \approx 5.3 \Omega_{b}$ [20]. A key ingredient of the computation is the baryon asymmetry. Its value measured in cosmic microwave background (CMB) and Big Bang nucleosynthesis $(\mathrm{BBN})$ is $Y_{B}=n_{B} / s=0.8 \times 10^{-10}$. The $\mathrm{DM}$ abundance is reproduced (using $M_{\mathcal{S}}=1.2 \mathrm{GeV}$ for definiteness) for

\footnotetext{
${ }^{1}$ We thank M. Karliner, A. Francis, and J. Green for discussions.
}

$$
\frac{Y_{\mathcal{S}}}{Y_{B}}=\frac{\Omega_{\mathrm{DM}}}{\Omega_{B}} \frac{M_{p}}{M_{\mathcal{S}}} \approx 4.2
$$

Thereby the baryon asymmetry before $\mathcal{S}$ decoupling must be

$$
Y_{B \mathcal{S}}=Y_{B}+2 Y_{\mathcal{S}} \approx 9.3 Y_{B} \text {. }
$$

One needs to evolve a network of Boltzmann equations for the main hadrons: $p, n, \Lambda, \Sigma^{0}, \Sigma^{+}, \Sigma^{-}, \Xi^{0}, \Xi^{-}$, and $\mathcal{S}$. Strange baryons undergo weak decays with lifetimes $\tau \sim 10^{-10} \mathrm{~s}$, a few orders of magnitude faster than the Hubble time. This means that such baryons stay in thermal equilibrium. We thereby first compute the thermal equilibrium values taking into account the baryon asymmetry. Thermal equilibrium implies that the chemical potentials $\mu(T)$ satisfy

$$
\mu_{b}=\mu_{S} / 2, \quad b=\{p, n, \Lambda, \ldots\} .
$$

Their overall values are determined imposing that the total baryon asymmetry equals

$$
\sum_{b} \frac{n_{b}^{\mathrm{eq}}-n_{\bar{b}}^{\mathrm{eq}}}{s}+2 \frac{n_{\mathcal{S}}^{\mathrm{eq}}-n_{\overline{\mathcal{S}}}^{\mathrm{eq}}}{s}=Y_{B \mathcal{S}}
$$

The equilibrium values can be analytically computed in Boltzmann approximation (which becomes exact in the nonrelativistic limit)

$$
n_{i}^{\mathrm{eq}}=g_{i} \frac{M_{i}^{2} T}{2 \pi^{2}} \mathrm{~K}_{2}\left(\frac{M_{i}}{T}\right) e^{ \pm \mu_{i} / T}
$$

where the $+(-)$ holds for (anti)particles. We then obtain the abundances in thermal equilibrium plotted in Fig. 1, 
assuming $M_{\mathcal{S}}=1.2 \mathrm{GeV}$. We see that the desired $\mathcal{S}$ abundance is reproduced if the interactions that form/destroy $\mathcal{S}$ decouple at $T_{\mathrm{dec}} \approx 25 \mathrm{MeV}$. This temperature is so low that baryon antiparticles have negligible abundances, and computations can more simply be done neglecting antiparticles. ${ }^{2}$ Then, the desired decoupling temperature is simply estimated imposing $n_{\mathcal{S}}^{\mathrm{eq}} / n_{p}^{\mathrm{eq}} \sim Y_{B \mathcal{S}} e^{\left(2 M_{p}-M_{\mathcal{S}}\right) / T} \sim 1$, and it decreases if $M_{\mathcal{S}}$ is heavier:

$$
\left.T_{\text {dec }}\right|_{\text {desired }} \approx \frac{2 M_{p}-M_{\mathcal{S}}}{\left|\ln Y_{B \mathcal{S}}\right|} \approx 89 \mathrm{MeV}-0.048 M_{\mathcal{S}}
$$

To compute the decoupling temperature, we consider the three different kinds of processes that can lead to the formation of $\mathcal{S}$ :

(1) Strong interactions of two heavier QCD hadrons that contain the needed two $s$ quarks. One example is $\Lambda \Lambda \leftrightarrow \mathcal{S} X$, where $X$ denotes pions. These are doubly Boltzmann suppressed by $e^{-2 m_{s} / T}$ at temperatures $T<m_{s}$.

(2) Strong interactions of one heavier strange hadron and weak $\Delta S=1$ interactions that form the other $s$ (as $d \bar{u} \rightarrow s \bar{u}$ ) from lighter hadrons. One example is $p \Lambda \leftrightarrow \mathcal{S} X$. These are singly Boltzmann suppressed by $e^{-m_{s} / T}$ and by $G_{\mathrm{F}}^{2} \Lambda_{\mathrm{QCD}}^{4} \sim 10^{-10}$.

(3) Double-weak interactions that form two $s$ quarks starting from lighter hadrons. One example is $p p \rightarrow \mathcal{S} X$. These are doubly suppressed by $\left(G_{\mathrm{F}}^{2} \Lambda_{\mathrm{QCD}}^{4}\right)^{2}$.

At $T \sim 25 \mathrm{MeV}$ the abundance of strange hadrons is still large enough that QCD processes dominate over EW processes: interactions that form and destroy $\mathcal{S}$ proceed dominantly through QCD collisions of strange hadrons,

$$
\Lambda \Lambda, n \Xi^{0}, p \Xi^{-}, \Sigma^{+} \Sigma^{-} \leftrightarrow \mathcal{S} X
$$

\footnotetext{
${ }^{2}$ Let us consider, e.g., the process $\Lambda+\Lambda \leftrightarrow \mathcal{S}+X$ where $X$ denotes other SM particles that do not carry the baryon asymmetry, such as pions. Thermal equilibrium of the above process implies$$
\frac{n_{\mathcal{S}}}{n_{\Lambda}^{2}}=\frac{n_{S}^{\mathrm{eq}}}{n_{\Lambda}^{\mathrm{eq} 2}}=\frac{g_{\mathcal{S}}}{g_{\Lambda}^{2}}\left(\frac{2 \pi M_{\mathcal{S}}}{M_{\Lambda}^{2} T}\right)^{3 / 2} e^{\left(2 M_{\Lambda}-M_{S}\right) / T} .
$$

Inserting $n_{\Lambda} \sim n_{p} e^{\left(M_{p}-M_{\Lambda}\right) / T}$ with $n_{p} \approx Y_{B \mathcal{S} S}$ gives

$$
\frac{n_{\mathcal{S}}}{n_{p}} \sim \frac{n_{\Lambda}^{2}}{n_{p}} e^{\left(2 M_{\Lambda}-M_{\mathcal{S}}\right) / T} \sim Y_{B \mathcal{S}} e^{\left(2 M_{p}-M_{\mathcal{S}}\right) / T} .
$$

Namely, $n_{\mathcal{S}} \ll n_{p}$ at large $T ; n_{\mathcal{S}} \gg n_{p}$ at low $T$. A DM abundance comparable to the baryon abundance is obtained only if reactions that form $\mathcal{S}$ decouple at the $T$ in Eq. (17).
}

where $X$ can be a $\pi^{0}$ or a $\gamma$, as preferred by approximate isospin conservation. The $\Lambda$ can be substituted by the $\Sigma^{0}$.

Defining $z=M_{\mathcal{S}} / T$ and $Y_{p}=n_{p} / s$, the Boltzmann equation for the $\mathcal{S}$ abundance is

$$
s H z \frac{d Y_{\mathcal{S}}}{d z}=\gamma_{\mathcal{S}}^{\mathrm{eqb}}\left[\frac{Y_{B}^{2}}{Y_{B}^{\mathrm{eqb} 2}}-\frac{Y_{\mathcal{S}}}{Y_{\mathcal{S}}^{\mathrm{eq}}}\right]
$$

where the superscript "eqb" denotes thermal equilibrium at fixed baryon asymmetry and $Y_{B}$ is summed over all baryons but the dibaryon $\mathcal{S}$. A second equation for $Y_{B}$ is not needed, given that the baryon number is conserved: $\left(Y_{B}-Y_{\bar{B}}\right)+2\left(Y_{\mathcal{S}}-Y_{\overline{\mathcal{S}}}\right)=Y_{B \mathcal{S}}$. Furthermore, $Y_{\overline{\mathcal{S}}}$ is negligible, and $Y_{\bar{B}}$ is negligible around decoupling. The $\mathcal{S}$ production rate is obtained after summing over all $b b^{\prime} \leftrightarrow$ $\mathcal{S} X$ processes of Eq. (18). In the nonrelativistic limit the interaction rate gets approximated as

$$
2 \gamma_{S}^{\mathrm{eqb}} \stackrel{T \ll}{\simeq} \sum_{b, b^{\prime}} n_{b}^{\mathrm{eqb}} n_{b^{\prime}}^{\mathrm{eqb}}\left\langle\sigma_{b b^{\prime}} v_{\mathrm{rel}}\right\rangle^{\mathrm{eqb}}
$$

The opposite process is more conveniently written in terms of the $\mathcal{S}$ breaking width defined by $\gamma_{\mathcal{S}}^{\text {eqb }}(T)=n_{S}^{\text {eqb }} \Gamma_{\mathcal{S}}^{\text {eqb }}$ and given by

$$
\Gamma_{S}^{\mathrm{eqb}}=\sum_{b, b^{\prime}} \frac{n_{b}^{\mathrm{eqb}} n_{b^{\prime}}^{\mathrm{eqb}}}{2 n_{S}^{\mathrm{eq}}}\left\langle\sigma_{b b^{\prime}} v_{\text {rel }}\right\rangle_{\text {eqb }} .
$$

This gets Boltzmann suppressed at $T \lesssim M_{\Lambda}-M_{p} \sim m_{s}$, when hyperons disappear from the thermal plasma. Assuming $Y_{\mathcal{S}} \lesssim Y_{B}$, the Boltzmann equation is approximatively solved by $Y_{\mathcal{S}} \sim Y_{\mathcal{S}}^{\mathrm{eqb}}$ evaluated at the decoupling epoch where $\Gamma_{\mathcal{S}}^{\mathrm{eqb}} \sim H$, which corresponds to $T_{\mathrm{dec}} \sim m_{s} / \ln Y_{B}$. This leads to the estimated final abundance

$$
\frac{Y_{\mathcal{S}}}{Y_{B}} \sim Y_{B}\left(M_{\mathrm{Pl}} T_{\mathrm{dec}} \sigma_{\mathcal{S}}\right)^{\frac{2 M_{p}-M_{\mathcal{S}}}{2 M_{\Lambda}-M_{\mathcal{S}}}}
$$

The fact that $\mathcal{S}$ is in thermal equilibrium down to a few tens of $\mathrm{MeV}$ means that whatever happens at higher temperatures gets washed out. Notice the unusual dependence on the cross section for $\mathcal{S}$ formation: increasing it delays the decoupling, increasing the $\mathcal{S}$ abundance.

Figure 2 shows the numerical result for the relic $\mathcal{S}$ abundance, computed by inserting in the Boltzmann equation a $s$-wave $\sigma_{b b^{\prime}} v_{\text {rel }}=\sigma_{0}$, varied around $1 / \mathrm{GeV}^{2}$. The cosmological DM abundance is reproduced for $M_{\mathcal{S}} \approx 1.2 \mathrm{GeV}$, while a large $M_{\mathcal{S}}$ gives a smaller relic abundance. Bound-state effects at BBN negligibly affect the result and, in particular, do not allow one to reproduce the DM abundance with a heavier $M_{\mathcal{S}} \approx 1.8 \mathrm{GeV}$.

We conclude this section with some sparse comments. Possible troubles with bounds from direct detection have 


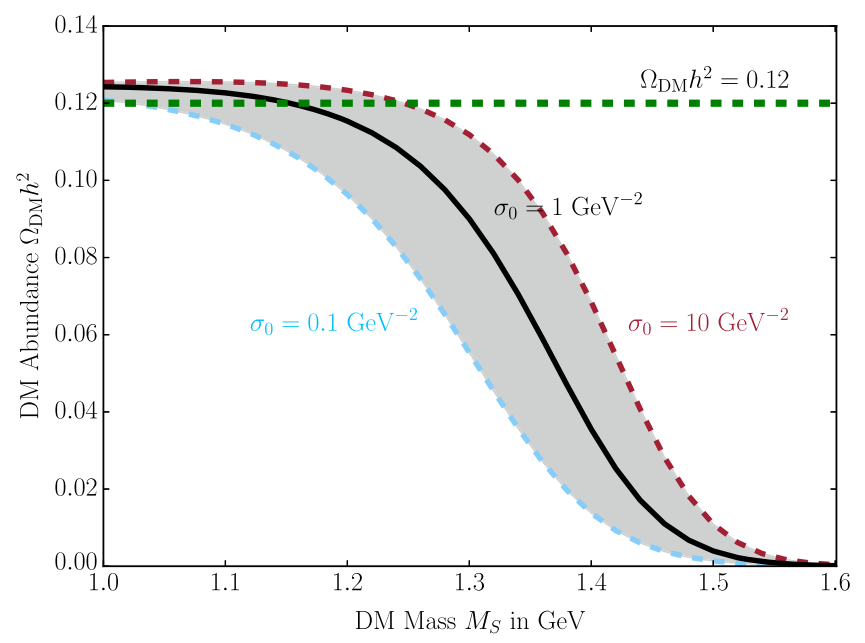

FIG. 2. Thermal hexaquark abundance within the SM with the baryon asymmetry in Eq. (11).

been pointed out in $[4,21,22]$ : a DM velocity somehow smaller than the expected one can avoid such bounds reducing the kinetic energy available for direct detection. Using a target made of antimatter (possibly in the upper atmosphere) would give a sharp annihilation signal, although with small rates. The magnetic dipole interaction of $\mathcal{S}$ does not allow one to explain the recent $21 \mathrm{~cm}$ anomaly along the lines of [23] (an electric dipole would be needed). The interactions of DM with the baryon/photon fluid may alter the evolution of cosmological perturbations leaving an imprint on the matter power spectrum and the CMB. However, they are not strong enough to produce significant effects. The $\mathcal{S}$ particle is electrically neutral and has spin zero, such that its coupling to photons is therefore suppressed by powers of the QCD scale [3]. So elastic scattering of $\mathcal{S}$ with photons is not cosmologically relevant.

A light $\mathcal{S}$ would affect neutron stars, as they are expected to contain $\Lambda$ particles, made stable by the large Fermi surface energy of neutrons. Then, $\Lambda \Lambda \rightarrow \mathcal{S}$ would give a loss of pressure, possibly incompatible with the observed existence of neutron stars with mass $2.0 M_{\text {sun }}$ [1]. However, we cannot exclude $\mathcal{S}$ on this basis, because production of $\Lambda$ hyperons poses a similar puzzle. $\mathcal{S}$ as DM could interact with cosmic ray $p$ giving and photon and other signals [24] and would be geometrically captured in the Sun, possibly affecting helioseismology. ${ }^{3}$

In the next section we discuss the main problem, which seems to exclude $\mathcal{S}$ as DM.

\section{Super-Kamiokande bound on nuclear stability}

Two nucleons $N=\{n, p\}$ inside a nucleus $\mathcal{N}$ can make a double weak decay into $\mathcal{S}$, emitting $\pi, \gamma$, or $e$ [2]. This is best probed by Super-Kamiokande (SK), which contains $\sim 8 \times 10^{32}$ oxygen nuclei. No dedicated search for

\footnotetext{
${ }^{3}$ We thank M. Pospelov for suggesting these ideas.
}

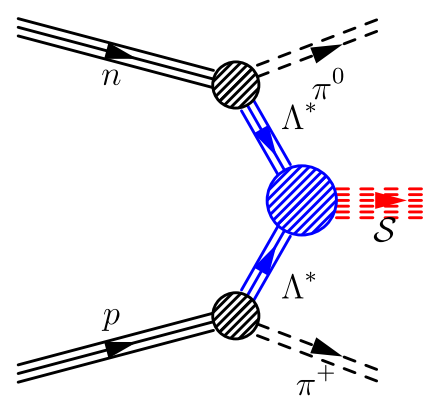

FIG. 3. Sample diagram that dominates nucleon decay into $\mathcal{S}$ inside nuclei. The initial state can also be $n n$ or $p p$.

${ }^{16} \mathrm{O}_{8} \rightarrow \mathcal{N}^{\prime} \mathcal{S} X$ (where $X$ can be one or two $\pi, \gamma, e$ and $\mathcal{N}^{\prime}$ can be ${ }^{14} \mathrm{O}_{8},{ }^{14} \mathrm{~N}_{7},{ }^{14} \mathrm{C}_{6}$, depending on the charge of $X$ ) has been performed, ${ }^{4}$ but a very conservative limit

$$
\tau\left({ }^{16} \mathrm{O}_{8} \rightarrow \mathcal{N}^{\prime} \mathcal{S} X\right) \gtrsim 10^{26} \mathrm{yr}
$$

is obtained by requiring the rate of such transitions to be smaller than the rate of triggered background events in SK, which is about $10 \mathrm{~Hz}$ [27]. A more careful analysis would likely improve this bound by 3 orders of magnitude [2]

The amplitude for the formation of $\mathcal{S}$ is reasonably dominated by the sample diagram in Fig. 3: doubly weak production of two virtual strange $\Lambda^{*}$ baryons (e.g., through $p \rightarrow \pi^{+} \Lambda^{*}$ and $n \rightarrow \pi^{0} \Lambda^{*}$; at quark level $u \rightarrow s \bar{d} u$ and $d \rightarrow s \bar{u} u$ ), followed by the strong process $\Lambda^{*} \Lambda^{*} \rightarrow \mathcal{S}$,

$$
\mathcal{M}_{N N \rightarrow \mathcal{S} X} \approx \mathcal{M}_{N N \rightarrow \Lambda^{*} \Lambda^{*} X} \times \mathcal{M}_{\Lambda^{*} \Lambda^{*} \rightarrow \mathcal{S}}
$$

The predicted lifetime is then obtained as $[2]^{5}$

$$
\begin{aligned}
& \tau\left(\mathcal{N} \rightarrow \mathcal{N}^{\prime} \mathcal{S} X\right) \\
& \quad \simeq \frac{\mathrm{yr}}{|\mathcal{M}|_{\Lambda^{*} \Lambda^{*} \rightarrow \mathcal{S}}^{2}} \times\left\{\begin{array}{lll}
3 & \text { if } M_{\mathcal{S}} \lesssim 1.74 \mathrm{GeV}, \\
10^{5} & \text { if } 1.74 \mathrm{GeV} \lesssim M_{\mathcal{S}} \lesssim 1.85 \mathrm{GeV},
\end{array}\right.
\end{aligned}
$$

where the smaller value holds if $\mathcal{S}$ is so light that the decay can proceed through real $\pi$ or $\pi \pi$ emission, while the longer lifetime is obtained if instead only lighter $e^{+} \nu$ or $\gamma$ can be emitted.

The key factor is the dimensionless matrix element $\mathcal{M}_{\Lambda^{*} \Lambda^{*} \rightarrow \mathcal{S}}$ for the transition $\Lambda^{*} \Lambda^{*} \rightarrow \mathcal{S}$ inside a nucleus,

\footnotetext{
${ }^{4}$ SK searched for dinucleon decays into pions [25] and leptons [26] and obtained bounds on the lifetime around $\sim 10^{32}$ years. However, these bounds are not directly applicable to ${ }^{16} \mathrm{O}_{8} \rightarrow$ $\mathcal{N}^{\prime} \mathcal{S} X$ where the invisible $\mathcal{S}$ takes away most of the energy reducing the energy of the visible pions and charged leptons, in contrast to what is assumed in $[25,26]$.

${ }^{5} \mathrm{~A}$ numerical factor of 1440 due to spin and flavor effects has already been factored out from $|\mathcal{M}|_{\Lambda^{*} \Lambda^{*} \rightarrow \mathcal{S}}^{2}$ here and in the following. Note also that the threshold $2 M_{N}-M_{\pi}=1.74 \mathrm{GeV}$ neglects the small difference in binding energy between $\mathcal{N}$ and $\mathcal{N}^{\prime}$.
} 
which we now discuss. Following [2], we assume that the initial state wave function can be factorized into wave functions of the two $\Lambda^{*}$ baryons and a relative wave function $\psi_{\text {nuc }}(\vec{a})$ for the separation $\vec{a}$ between the center of mass of the $\Lambda^{*}$ 's. The matrix element is given by the wave-function overlap

$$
\mathcal{M}_{\Lambda^{*} \Lambda^{*} \rightarrow \mathcal{S}}=\int \psi_{\mathcal{S}}^{*}\left(\vec{\rho}^{a}, \vec{\lambda}^{a}, \vec{\rho}^{b}, \vec{\lambda}^{b}, \vec{a}\right) \psi_{\Lambda^{*}}\left(\vec{\rho}^{a}, \vec{\lambda}^{a}\right) \psi_{\Lambda^{*}}\left(\vec{\rho}^{b}, \vec{\lambda}^{b}\right) \psi_{\mathrm{nuc}}(\vec{a}) d^{3} a d^{3} \rho^{a} d^{3} \rho^{b} d^{3} \lambda^{a} d^{3} \lambda^{b}
$$

Here, $\vec{\rho}^{a, b}, \vec{\lambda}^{a, b}$ are center-of-mass coordinates that parametrize the relative positions of the quarks within each $\Lambda^{*}$. Using the Isgur-Karl (IK) model [28] the wave functions for the quarks inside the $\Lambda^{*}$ and inside the $\mathcal{S}$ are approximated by

$$
\begin{gathered}
\psi_{\Lambda^{*}}(\vec{\rho}, \vec{\lambda})=\left(\frac{1}{r_{N} \sqrt{\pi}}\right)^{3} \exp \left[-\frac{\vec{\rho}^{2}+\vec{\lambda}^{2}}{2 r_{N}^{2}}\right] \\
\psi_{\mathcal{S}}\left(\vec{\rho}^{a}, \vec{\lambda}^{a}, \vec{\rho}^{b}, \vec{\lambda}^{b}, \vec{a}\right)=\left(\frac{3}{2}\right)^{3 / 4}\left(\frac{1}{r_{\mathcal{S}} \sqrt{\pi}}\right)^{15 / 2} \exp \left[-\frac{\vec{\rho}^{a 2}+\vec{\lambda}^{a 2}+\vec{\rho}^{b 2}+\vec{\lambda}^{b 2}+\frac{3}{2} \vec{a}^{2}}{2 r_{\mathcal{S}}^{2}}\right],
\end{gathered}
$$

where $r_{N}$ and $r_{\mathcal{S}}$ are the radii of the nucleons, respectively, of $\mathcal{S}{ }^{6}$ Performing all integrals except the final integral over $a \equiv|\vec{a}|$ gives

$$
|\mathcal{M}|_{\Lambda^{*} \Lambda^{*} \rightarrow \mathcal{S}}=\frac{1}{2}\left(\frac{3}{2}\right)^{3 / 4}\left(\frac{2 r_{N} r_{\mathcal{S}}}{r_{N}^{2}+r_{\mathcal{S}}^{2}}\right)^{6}\left(\frac{1}{r_{\mathcal{S}} \sqrt{\pi}}\right)^{3 / 2} \int d a 4 \pi a^{2} e^{-3 a^{2} / 4 r_{\mathcal{S}}^{2}} \psi_{\text {nuc }}(a)
$$

As shown in Fig. 5 below (and as discussed in [2]), if $r_{\mathcal{S}}$ is not much smaller than $r_{N}$, the overlap integral is not very much suppressed and $\tau\left({ }^{16} \mathrm{O}_{8} \rightarrow \mathcal{N}^{\prime} \mathcal{S} X\right)$ is tens of orders of magnitude below the experimental limit and is clearly excluded. This conclusion is independent of the form of $\psi_{\text {nuc }}$.

However, if $r_{\mathcal{S}}$ were a few times smaller than $r_{N}-\mathrm{a}$ possibility that seems unlikely due to diquark repulsions (see, e.g., [19]) but cannot firmly be excluded-then $\tau\left({ }^{16} \mathrm{O}_{8} \rightarrow \mathcal{N}^{\prime} \mathcal{S} X\right)$ is extremely sensitive to the probability of the overlap of two nucleons inside the oxygen core at very small distances (less than, say, $0.5 \mathrm{fm}$ ). The wave function of nucleon pairs $\psi_{\text {nuc }}$ at such small distances has not been probed experimentally. In fact, at such small distances nucleons are not the appropriate degrees of freedom. ${ }^{7}$ Thus, for a very small $\mathcal{S}$ one can only make an educated guess of $\tau\left({ }^{16} \mathrm{O}_{8} \rightarrow \mathcal{N}^{\prime} \mathcal{S} X\right)$, since the form of $\psi_{\text {nuc }}$ is uncertain. Nevertheless, we will show in the

\footnotetext{
${ }^{6}$ One should be aware that the IK model has serious shortcomings. One issue is that it is a nonrelativistic model-an assumption that is problematic in particular for small $\mathcal{S}$. Another problem is that the value of $r_{N}$ that gives a good fit to the lowest lying $\frac{1}{2}^{+}$and $\frac{3}{2}^{+}$baryons $-r_{N}=0.49 \mathrm{fm}$-is smaller than the charge radius of the proton: $r_{N}=0.87 \mathrm{fm}$. Therefore we consider both $r_{N}=0.49 \mathrm{fm}$ and $r_{N}=0.87 \mathrm{fm}$, as done in [2].

${ }^{7}$ Data indicate that about $20 \%$ of the nucleons form $p n$ pairs so close (about $1 \mathrm{fm}$ ) that the local density reaches the nucleon density (about 2.5 times larger than the nuclear density) and thus that the quark structure of nucleons starts becoming relevant already at $a \sim 1 \mathrm{fm}[29]$.
}

following that for a reasonable form of $\psi_{\text {nuc }}$ a stable $\mathcal{S}$ is excluded even if it were very small.

Numerical computations of the ground-state wave functions of nuclei, including ${ }^{16} \mathrm{O}_{8}$, have been performed, e.g., in [5]. The quantity that determines $\psi_{\text {nuc }}$ is the two-nucleon point density $\rho_{N N}$, defined in Eq. (58) of [5]. We obtain $\rho_{N N}(a)$ by interpolating the data given in [5] and adding the constraint $\rho_{N N}(0)=0$, which is a conservative assumption for our purposes since $\rho_{N N}(0) \neq 0$ would lead to a larger matrix element. There are 28 neutron-neutron pairs and 64 proton-neutron pairs in ${ }^{16} \mathrm{O}_{8}$ so one has $\int d a 4 \pi a^{2} \rho_{n n}(a)=$ 28 and $\int d a 4 \pi a^{2} \rho_{p n}(a)=64$. We therefore define the wave functions

$\psi_{\text {nuc }}^{n n}(a)=\sqrt{\rho_{n n}(a) / 28}, \quad \psi_{\text {nuc }}^{p n}(a)=\sqrt{\rho_{p n}(a) / 64}$.

These wave functions are plotted in Fig. 4, together with the Miller-Spencer (MS) and the Brueckner-Bethe-Goldstone (BBG) wave function used in [2]. The BBG wave functions assume a hard repulsive core between nucleons such that $\psi_{\text {nuc }}$ vanishes at $a<r_{\text {core }}$. We take $r_{\text {core }}=0.5 \mathrm{fm}$ for illustration. This is not realistic but allows one to see what kind of nuclear wave function would sufficiently suppress the rate of $\mathcal{S}$ formation in nuclei, if $\mathcal{S}$ is small enough. The resulting $|\mathcal{M}|_{\Lambda^{*} \Lambda^{*} \rightarrow \mathcal{S}}^{2}$ is plotted in Fig. 5, again compared to that obtained using the Miller-Spencer and BBG wave functions. 


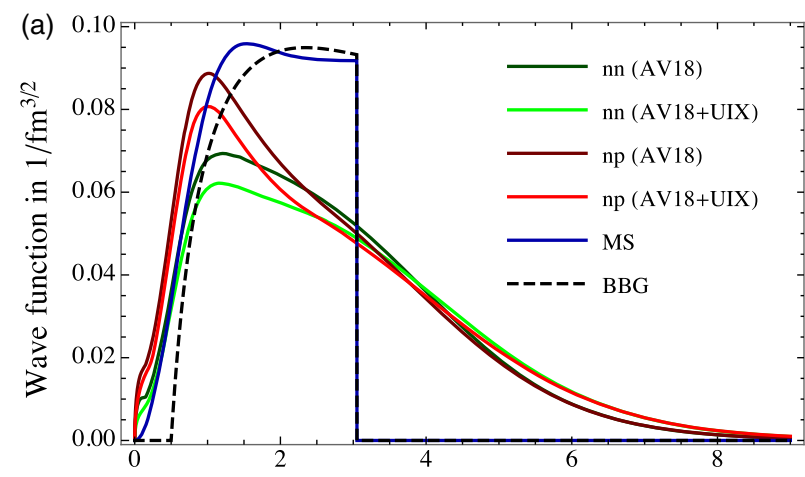

Distance between nucleons in $\mathrm{fm}$

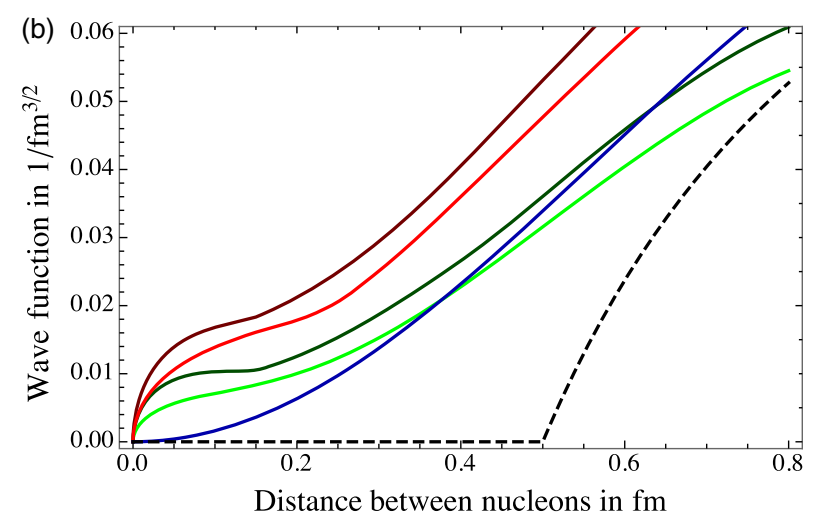

FIG. 4. Wave functions $\psi_{\text {nuc }}(a)$ as a function of the relative distance $a$ between two nucleons in ${ }^{16} \mathrm{O}_{8}$. The darker (lighter) green curve shows $\psi_{\text {nuc }}^{n n}$, obtained from [5], using the AV18 (AV18+UIX) potential; the darker (lighter) red curve shows $\psi_{\text {nuc }}^{n p}$ using the AV18 (AV18 +UIX) potential; the blue curve is the MS wave function used in [2] and the dashed black curve is the BBG wave function with hard core radius $r_{\text {core }}=0.5 \mathrm{fm}$. Left: Wave functions over the entire range. Right: Zoom-in plot of the region relevant for our calculation.

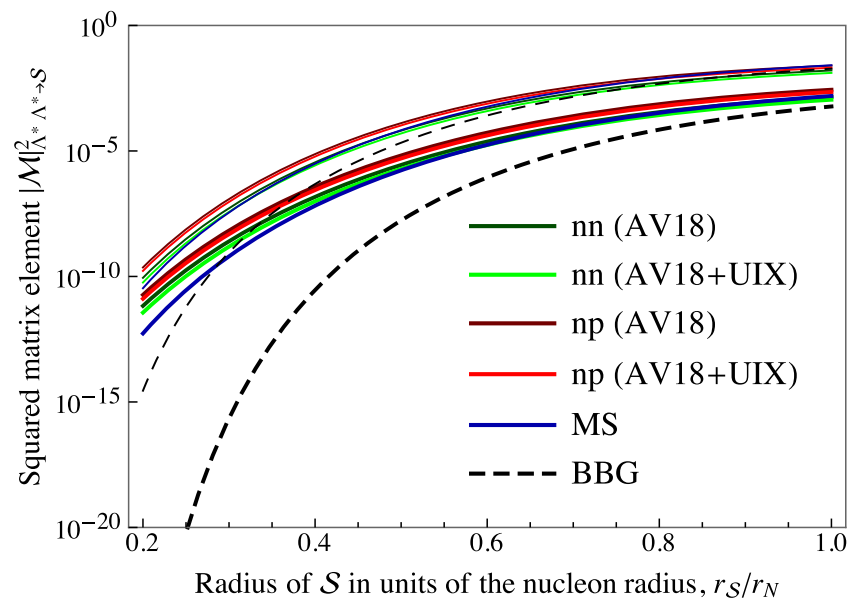

FIG. 5. The dimensionless squared matrix element for nuclear decay into $\mathcal{S},|\mathcal{M}|_{\Lambda^{*} \Lambda^{*} \rightarrow \mathcal{S}}^{2}$, as a function of the $\mathcal{S}$ radius $r_{\mathcal{S}}$ in units of the nucleon radius $r_{N}$, using different nuclear wave functions. The color coding, defined in Fig. 4, refers to the nuclear wave functions used. The thinner (thicker) curves assume $r_{N}=$ $0.87 \mathrm{fm}\left(r_{N}=0.49 \mathrm{fm}\right)$. The Super-Kamiokande bound would be evaded for $|\mathcal{M}|_{\Lambda^{*} \Lambda^{*} \rightarrow \mathcal{S}}^{2} \lesssim 10^{-20}\left(10^{-25}\right)$ for $M_{\mathcal{S}}>1.74 \mathrm{GeV}$ (for $M_{\mathcal{S}}<1.74 \mathrm{GeV}$ ).

The resulting matrix elements from the MS wave function qualitatively agree to what is obtained using the wave functions extracted from [5]. By contrast, the matrix element using the BBG wave function with hard core radius $r_{\text {core }}=0.5 \mathrm{fm}$ is very much suppressed, especially if $\mathcal{S}$ is small. The reason is that, according to the assumption of a hard core repulsive potential, the nucleons cannot get close enough to form the small state $\mathcal{S}$. Since we do not consider a $\psi_{\text {nuc }}(a)$ that vanishes for $a \lesssim 0.5$ fm realistic, we conclude that a stable $\mathcal{S}$ is excluded.

Weaker bounds on $\mathcal{S}$ production are obtained considering baryons containing $\Lambda$ 's.

\section{DM AS BLACK HOLES TRIGGERED BY HIGGS FLUCTUATIONS}

We here present the technical computations relative to the mechanism anticipated in the Introduction. The SM potential is summarized in Sec. III A. In Sec. III B we outline the mechanism that generates black holes. Section III C studies the generation of Higgs inhomogeneities. Postinflationary dynamics is studied in Sec. III D. The formation of black holes is considered in Sec. III E. The viability of a critical assumption is discussed in Sec. III F.

\section{A. The Higgs effective potential}

The effective potential of the canonically normalized Higgs field during inflation with Hubble constant $H_{0}$ is

$$
V_{\text {eff }}(h) \approx \frac{\lambda_{\text {eff }}(h)}{4} h^{4}-6 \xi_{H} H_{0}^{2} h^{2}+V_{0},
$$

at $h \gg 174 \mathrm{GeV}$. Here $\lambda_{\text {eff }}$ is the effective quartic coupling computed including quantum corrections. The second mass term in $V_{\text {eff }}(h)$ can be generated by various different sources [8]. We consider the minimal source: a Higgs coupling to gravity, $\mathcal{L}_{\xi}=-\xi_{H} R h^{2} / 2$, with Ricci scalar $R=-12 H_{0}^{2}$ during inflation. Finally, during inflation the effective potential in Eq. (31) is augmented by the vacuum energy associated with the inflaton sector, $V_{0}=3 \bar{M}_{\mathrm{Pl}}^{2} H_{0}^{2}$, where $\bar{M}_{\mathrm{Pl}} \simeq$ $2.435 \times 10^{18} \mathrm{GeV}$ is the reduced Planck mass.

We implement the renormalization group (RG) improvement of the effective potential at next-to-next-to-leading order (NNLO) precision: running the SM parameters at three loops and including two-loop quantum corrections to the effective potential. We consider fixed values of $\alpha_{3}\left(M_{Z}\right)=0.1184$ and $M_{h}=125.09 \mathrm{GeV}$, and we vary the main uncertain parameter, the top mass, in the interval $M_{t}=172.5 \pm 0.5 \mathrm{GeV}$ [30]. In Fig. 6(a) we show the resulting $\lambda_{\text {eff }}(h)$ as a function of $h$. 

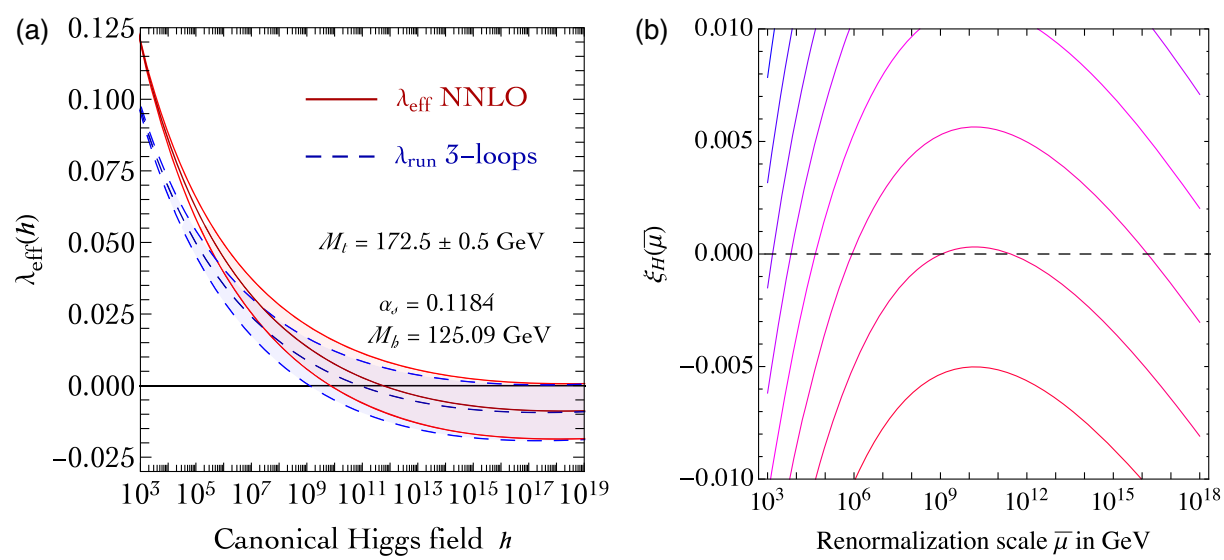

FIG. 6. Left: RGE running of $\lambda_{\text {eff }}$ (solid red lines) obtained by changing the top mass in its $3 \sigma$ interval defined by $M_{t}=172.5 \pm 0.5 \mathrm{GeV}$. For comparison, we show the running of $\lambda$ at three loops (dashed blue lines) without including the Coleman-Weinberg corrections. Right: RGE running of a small $\xi_{H}$.
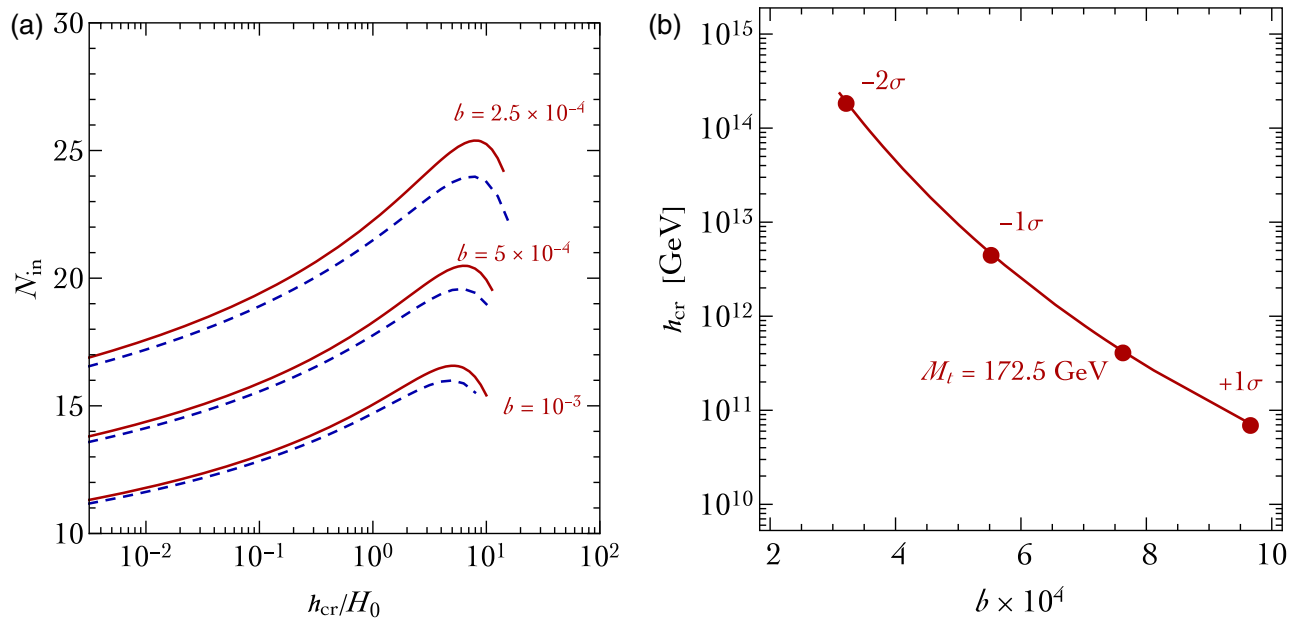

FIG. 7. Left: Number of $e$-folds $N_{\text {in }}$ at the beginning of the Higgs fall that gives the maximal $h_{\text {end }}$ rescued by the reheating temperature. This is computed as a function of the Hubble constant during inflation, for three different values of the uncertain parameter $b$ that approximates the Higgs potential. Continuous (dashed) curves correspond to $\xi_{H}=0\left(-10^{-3}\right)$. Right: SM values of $b$ and of the position $h_{\mathrm{cr}}$ of the top of the SM potential as a function of the top mass.

The nonminimal coupling to gravity $\xi_{H}$ receives SM quantum corrections encoded in its renormalization group equation (RGE), which induce $\xi_{H} \neq 0$ even starting from $\xi_{H}=0$ at some energy scale. The RGE running of small values of $\xi_{H}(\bar{\mu})$ is shown in Fig. 6(b). As mentioned before, a nonzero $\xi_{H}$ can be considered as a proxy for an effective mass term during inflation. The latter, for instance, can be generated by a quartic interaction $\lambda_{h \phi}|H|^{2} \phi^{2}$ between the Higgs and the inflaton field or by the inflaton decay into SM particles during inflation. For these reasons, it makes sense to include $\xi_{H}$ as a free parameter in the analysis of the Higgs dynamics during inflation, at most with the theoretical bias that its size could be loop suppressed.

\section{Analytic approximation}

We will show precise numerical results for the SM case. However, the discussion is clarified by introducing a simple approximation that encodes the main features of the SM effective potential in Eq. (31),

$$
V_{\text {eff }}(h) \approx-b \log \left(\frac{h^{2}}{h_{\mathrm{cr}}^{2} \sqrt{e}}\right) \frac{h^{4}}{4}-6 \xi_{H} H_{0}^{2} h^{2},
$$

where $h_{\mathrm{cr}}$ is the position of the maximum of the potential with no extra mass term, $\xi_{H}=0$. The parameters $b$ and $h_{\mathrm{cr}}$ depend on the low-energy SM parameters such as the top mass: they can be computed by matching the numerical value of the Higgs effective potential at the gauge-invariant position of the maximum, $V_{\mathrm{eff}}\left(h_{\mathrm{cr}}\right)=b h_{\mathrm{cr}}^{4} / 8$. The result is shown in the right panel of Fig. 7.

Results will be better understood when presented in terms of the dimensionless parameters $b, \xi_{H}, h_{\mathrm{cr}} / H_{0}$, and $T / H_{0}$, where $T$ is the temperature, as they directly control 
the dynamics that we are going to study. The parameter $b$ controls the flatness of the potential beyond the potential barrier at $h_{\mathrm{cr}}$, with smaller $b$ corresponding to a flatter potential. The nonminimal coupling $\xi_{H}$ controls the effective Higgs mass during inflation. Finally, $\bar{M}_{\mathrm{Pl}} / H_{0}$ will set the reheating temperature in Eq. (35) and thus the position and size of the thermal barrier.

The position of the potential barrier-defined by the field value where the effective potential has its maximumstrongly depends on the value of the top mass, on the nonminimal coupling to gravity, and, after inflation, on the temperature of the thermal bath that provides an extra mass term. For $\xi_{H} \neq 0$, the maximum of the Higgs potential gets shifted from $h_{\mathrm{cr}}$ to

$$
h_{\max }=H_{0}\left[-\frac{b}{12 \xi_{H}} \mathcal{W}\left(\frac{-12 \xi_{H} H_{0}^{2}}{b h_{\mathrm{cr}}^{2}}\right)\right]^{-1 / 2},
$$

where $\mathcal{W}(z)$ is the product-log function defined by $z=\mathcal{W} e^{\mathcal{W}}$. The condition

$$
-12 \xi_{H} H_{0}^{2}>-\frac{b h_{\mathrm{cr}}^{2}}{e}
$$

must be satisfied; otherwise, the effective mass is too negative, and it erases the potential barrier, thus leading to a classical instability.

\section{The thermal potential}

After the end of inflation, the Higgs effective potential receives large thermal corrections from the SM bath at generic temperature $T$. The initial temperature of the thermal bath is fixed by the dynamics of reheating after inflation. We assume instantaneous reheating, as this is most efficient for rescuing the falling Higgs field. The reheating temperature is then given by

$$
T_{\mathrm{RH}}=\left(\frac{45}{4 \pi^{3} g_{*}}\right)^{1 / 4} M_{\mathrm{Pl}}^{1 / 2} H_{0}^{1 / 2}
$$

where $g_{*}=106.75$ is the number of SM degrees of freedom. After reheating, the Universe becomes radiation dominated, the Ricci scalar vanishes, and so does the contribution to the effective potential from the nonminimal Higgs coupling to gravity.

The effective Higgs potential at finite temperature is obtained adding an extra thermal contribution $V_{\mathrm{T}}$, which can be approximated as an effective thermal mass for the Higgs field, $M_{\mathrm{T}}^{2} \simeq 0.12 T^{2}$ (see, e.g., [10])

$$
\begin{aligned}
& V_{\mathrm{eff}}^{\mathrm{T}}(h) \approx-b \log \left(\frac{h^{2}}{h_{\mathrm{cr}}^{2} \sqrt{e}}\right) \frac{h^{4}}{4}+V_{\mathrm{T}}(h), \\
& V_{\mathrm{T}}(h) \approx \frac{1}{2} M_{\mathrm{T}}^{2} h^{2} e^{-h / 2 \pi T} .
\end{aligned}
$$

At $h \lesssim T$ we can neglect the exponential suppression in the thermal mass, and the maximum of the effective potential in Eq. (36) is given by

$$
h_{\max }^{\mathrm{T}}=M_{\mathrm{T}}\left[b \mathcal{W}\left(\frac{M_{\mathrm{T}}^{2}}{b h_{\mathrm{cr}}^{2}}\right)\right]^{-1 / 2} .
$$

\section{B. Outline of the mechanism}

During inflation, the Higgs field is subject to quantum fluctuations. Depending on the value of $H_{0}$, these quantum fluctuations could lead the Higgs beyond the barrier and make it roll toward Planckian values. If $T_{\mathrm{RH}}$ is high enough and $h$ is not too far, thermal corrections can "rescue" the Higgs, bringing it back to the origin [10]. The mechanism relies on a tuning such that the following situation occurs [8]:

(i) At $N_{\text {in }} \sim 20 e$-folds before the end of inflation, the Higgs background value $h$ is brought by quantum fluctuation to some $h_{\text {in }} \neq 0$. This configuration must be spatially homogeneous on an inflating local patch large enough to encompass our observable Universe today. We consider the de Sitter metric in flat slicing coordinates, $d s^{2}=-d t^{2}+a^{2}(t) d \vec{x}^{2}$. We will discuss later how precisely this assumption must be satisfied, as well as its plausibility.

(ii) When the classical evolution prevails over the quantum corrections, the Higgs field, starting from the initial position $h_{\text {in }}$, begins to slow roll down the negative potential. This condition reads

$$
\underbrace{\left|\frac{V_{\text {eff }}^{\prime}\left(h_{\text {in }}\right)}{3 H_{0}^{2}}\right|}_{\text {classical }}>\underbrace{\frac{c H_{0}}{2 \pi}}_{\text {quantum }},
$$

where $c$ is a constant of order 1, fixed to $c=1$ in [8]. We will explore what happens choosing $c=0.9$ or $c=1.1$. From this starting point $t_{\text {in }}$ on, the classical evolution of the background Higgs value is described by

$$
\ddot{h}_{\mathrm{cl}}+3 H_{0} \dot{h}_{\mathrm{cl}}+V_{\mathrm{eff}}^{\prime}\left(h_{\mathrm{cl}}\right)=0,
$$

where the subscript ${ }_{\mathrm{cl}}$ indicates that this is a classical motion. Dots indicate derivatives with respect to time $t$.

(iii) At the end of inflation, $N_{\text {end }}=0$, the Higgs is rescued by thermal effects. This happens if the value of the Higgs field $h_{\text {end }}$ at the end of inflation is smaller than the position of the thermal potential 
barrier at reheating, $h_{\mathrm{max}}^{T_{\mathrm{RH}}}$. A significant amount of $\mathrm{PBH}$ arises only if this condition is barely satisfied in all the Universe. This is why the homogeneity assumption in (i) is needed.

To compute condition (iii) we fix the initial value of the classical motion $h_{\text {in }}$ such that Eq. (38) is satisfied with $c=1$; next, we maximize the $h_{\text {end }}$ obtained solving Eq. (39) by tuning the amount of inflation where the fall happens, as parametrized by $N_{\text {in. }}$. The left panel of Fig. 7 shows the initial value $N_{\text {in }}$ obtained following this procedure as a function of $h_{\text {cr }}$ in units of $H_{0}$. Smaller values of $b$ (i.e., smaller values of $M_{t}$ ) imply a flattening of the potential, and the classical dynamics during inflation is slower. The right side of the curves is limited by the classicality condition in Eq. (38). A $\xi_{H}<0$ shifts the position of the potential barrier toward the limiting value $h_{\max }^{\mathrm{T}}$ in Eq. (37)_which does not depend on $\xi_{H}$-above which the rescue mechanism due to thermal effects is no longer effective: its net effect is to reduce the number of $e$-folds during which classical motion can happen (for fixed $b$ ).

We anticipate here the feature of $\mathrm{PBH}$ formation, which implies the restriction on the parameter space mentioned at point (i): the Higgs fall must start at least $N_{\text {in }} \sim 20 e$-folds before the end of inflation. The collapse of the mass inside the horizon $N e$-folds before the inflation end forms a $\mathrm{PBH}$ with mass (see also Sec. III E),

$$
M_{\mathrm{PBH}} \approx \frac{\bar{M}_{\mathrm{Pl}}^{2}}{H_{0}} e^{2 N}
$$

PBH must be heavy enough to avoid Hawking evaporation. The lifetime of a PBH with mass $M_{\mathrm{PBH}}$ due to Hawking radiation at Bekenstein-Hawking temperature $T_{\mathrm{BH}}=$ $1 /\left(8 \pi G_{N} M_{\mathrm{PBH}}\right)$ is

$$
\Gamma_{\mathrm{PBH}}^{-1} \approx 4 \times 10^{11}\left[\frac{\mathcal{F}\left(M_{\mathrm{PBH}}\right)}{15.35}\right]^{-1}\left(\frac{M_{\mathrm{PBH}}}{10^{13} \mathrm{~g}}\right)^{3} \mathrm{~s},
$$

where $\mathcal{F} \rightarrow 1$ at $M_{\mathrm{PBH}}>10^{17}$ g. BH heavier than $M_{\mathrm{PBH}}>$ $10^{15} \mathrm{~g}$ are cosmologically stable, and $\mathrm{BH}$ heavier than $M_{\mathrm{PBH}}>10^{16.5} \mathrm{~g}$ are allowed by bounds on Hawking radiation as a (significant fraction of) DM. Since $N<N_{\text {in }}$, imposing $M_{\mathrm{PBH}}>10^{16.5} \mathrm{~g}$ implies a conservative lower limit on $N_{\text {in }}$,

$N_{\text {in }}>\frac{1}{2} \ln \left[7.2 \times 10^{21} \frac{H_{0}}{\bar{M}_{\mathrm{Pl}}}\right]=18.3$ for $H_{0}=10^{-6} \bar{M}_{\mathrm{Pl}}$.

\section{Higgs fluctuations during inflation}

We now consider the evolution of Higgs perturbations during inflation. Expanding $h$ in Fourier space with comoving wave number $k,{ }^{8}$ the equation for the mode $\delta h_{k}$ takes the form

$$
\ddot{\delta} \dot{h}_{k}+3 H_{0} \dot{\delta h_{k}}+\frac{k^{2}}{a^{2}} \delta h_{k}+V_{\mathrm{eff}}^{\prime \prime}\left(h_{\mathrm{cl}}\right) \delta h=0
$$

where we neglected metric fluctuations. In terms of the number of $e$-folds $N$ and of the Mukhanov-Sasaki variable $u_{k} \equiv a \delta h_{k}$, it becomes

$\frac{d^{2} u_{k}}{d N^{2}}+\frac{d u_{k}}{d N}+\left(\frac{k^{2}}{a^{2} H_{0}^{2}}-2\right) u_{k}+\frac{V_{\mathrm{eff}}^{\prime \prime}\left(h_{\mathrm{cl}}\right)}{H_{0}^{2}} u_{k}=0$.

It is convenient to consider the evolution of the perturbation making reference to a specific moment before the end of inflation: at the initial value $N_{\text {in }}$ defined in Sec. III B. We recall that in our convention $N_{\text {end }}=0$ at the end of inflation. Equation (44) becomes

$$
\begin{gathered}
\frac{d^{2} u_{k}}{d N^{2}}-\frac{d u_{k}}{d N}+\left[\left(\frac{k}{a_{\mathrm{in}} H_{0}}\right)^{2} e^{2\left(N-N_{\mathrm{in}}\right)}-2\right] u_{k} \\
+\frac{V_{\mathrm{eff}}^{\prime \prime}\left(h_{\mathrm{cl}}\right)}{H_{0}^{2}} u_{k}=0 .
\end{gathered}
$$

In this form, the Mukhanov-Sasaki equation is particularly illustrative. Consider the evolution of the perturbation for a mode of interest $k$ that we fix compared to the reference value $a_{\text {in }} H_{0}$ at $t=t_{\text {in }}$. In particular, we consider the case of a mode $k$ that is subhorizon at the beginning of the classical evolution, that is, $k \gg a_{\text {in }} H_{0}$. From Eq. (45), we see that in the subsequent evolution with $N<N_{\text {in }}$ the exponential suppression will turn the mode from subhorizon to superhorizon.

We are now in the position to solve Eq. (45). To this end, we need boundary conditions for $u_{k}$ and its time derivative. We use the Bunch-Davies conditions at $N=N_{\text {in }}$ for modes that are subhorizon at the beginning of the classical evolution, $k / a_{\text {in }} H_{0} \geq 1$, and we treat the real and imaginary parts of $u_{k}$ separately since they behave as two independent harmonic oscillators for each comoving wave number $k$. At generic $e$-fold time $N$, the perturbation $\delta h_{k}$ is related to the Mukhanov-Sasaki variable $u_{k}$ by

$$
\left.k^{3 / 2} \frac{\delta h_{k}}{H_{0}}\right|_{N}=\left.\left(\frac{k}{a_{\mathrm{in}} H_{0}}\right) e^{N-N_{\text {in }}}\left(\sqrt{k} u_{k}\right)\right|_{N} .
$$

We show in the left panel of Fig. 8 our results for the time evolution of the classical background $h_{\mathrm{cl}}$ and the perturbation $\delta h_{k}$ (both real and imaginary parts) during the last 20 $e$-folds of inflation. As a benchmark value, we consider an initial subhorizon mode with $k / a_{\text {in }} H_{0}=100$. After a few

\footnotetext{
${ }^{8}$ The comoving wave number $k=|\vec{k}|$ is time independent, and it is related to the physical momentum via $k_{\text {phys }}=k / a(t)$, which decreases as the space expands.
} 

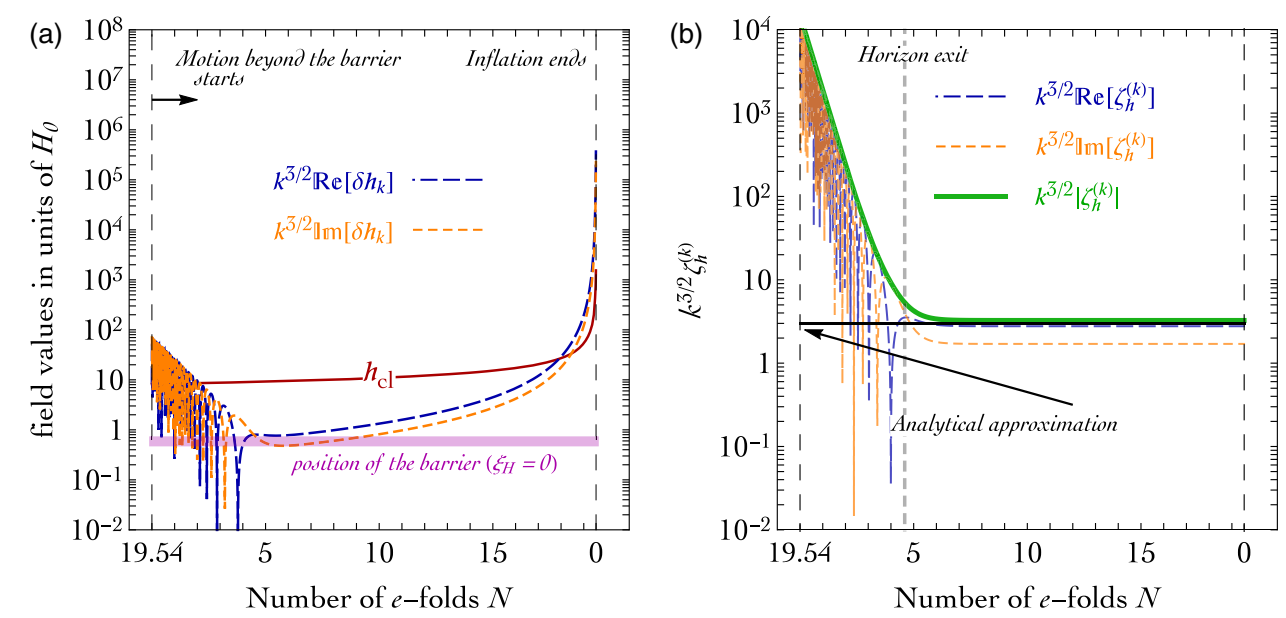

FIG. 8. Left: Sample evolution of the classical Higgs background ( $h_{\mathrm{cl}}$, red solid line) and of a perturbation with $k / a_{\text {in }} H_{0}=10^{2}$ (dashed lines). Right: Higgs curvature perturbation $\zeta_{h}^{(k)}$ during inflation. We compare the full numerical result with the analytical approximation [last term in Eq. (52), solid horizontal black line]. The vertical dashed gray line marks the instant of horizon exit. We use the analytical approximation in Eq. (32) with $h_{\mathrm{cr}}=410^{12} \mathrm{GeV}, b=0.09 /(4 \pi)^{2}$ (which corresponds to $M_{t}=172 \mathrm{GeV}$ ), and $H_{0}=10^{12} \mathrm{GeV}$.

$e$-folds of inflation, such a mode exits the horizon: oscillations stop, and from this point on, further evolution is driven by the time derivative of the classical background. This is a trivial consequence of the equations of motion on superhorizon scales. Differentiating Eq. (39) with respect to the cosmic time shows that $\dot{h}_{\mathrm{cl}}$ and $\delta h_{k}$ satisfy the same equation on superhorizon scales, and, therefore, they must be proportional, $\delta h_{k}=C(k) \dot{h}_{\mathrm{cl}}$ for $k \ll a H_{0} \quad$ [8]. The proportionality function $C(k)$ can be obtained by a matching procedure. Deep inside the horizon, in the limit $k \gg a H_{0}$, the Mukhanov-Sasaki variable $u_{k} \equiv a \delta h_{k}$ reproduces the preferred vacuum of a harmonic oscillator in flat Minkowski space, and we have, after introducing the conformal time $\tau$ as $d t=a d \tau, u_{k}=e^{-i k \tau} / \sqrt{2 k}$. Roughly matching the absolute value of the solutions at horizon crossing, we determine the absolute value of $C(k)$ as

$$
\left|\delta h_{k}\right|=\frac{H_{0}}{\sqrt{2 k^{3}} \dot{h}_{\mathrm{cl}}\left(t_{k}\right)} \dot{h}_{\mathrm{cl}}(t)
$$

where we indicate with $t_{k}$ the time of the horizon exit for the mode $k$-the time at which $k=a\left(t_{k}\right) H_{0} \equiv a_{k} H_{0}$ (equivalently, $-k \tau_{k}=1$ ). The number of $e$-folds at the horizon exit is given by

$$
\frac{k}{a_{\mathrm{in}} H_{0}}=e^{N_{\mathrm{in}}-N_{k}} .
$$

\section{Primordial curvature perturbations}

The primordial curvature perturbation $\zeta(\vec{x}, t)$ on uniform energy density slices $\rho$ is defined (at the linear order) by the perturbed line element $[31,32]$

$$
d s^{2}=a^{2}(t)[1+2 \zeta(\vec{x}, t)] \delta_{i j} d x^{i} d x^{j},
$$

and it is related to the (total) energy density perturbation $\delta \rho$ and to the curvature perturbation on a generic slicing $\Psi$ by the gauge invariant formula

$$
\zeta=\Psi+\mathcal{H} \frac{\delta \rho}{\rho^{\prime}}=\Psi+H \frac{\delta \rho}{\dot{\rho}},
$$

where we introduced the conformal time by means of $d t=$ $a d \tau$ and $\mathcal{H}=a H$ where $\mathcal{H}=a^{\prime} / a$ and prime ' indicates the derivative with respect to $\tau$. The virtue of this definition is that, on superhorizon scales, $\zeta$ is practically identical to the comoving curvature perturbation $\mathcal{R}$ defined on hypersurfaces of constant comoving time. Furthermore, on superhorizon scales $\zeta$ is conserved provided that the pressure perturbation is adiabatic. In conventional singlefield inflation models, purely adiabatic perturbations are generated due to quantum fluctuations of the single field driving inflation. The considered setup differs from a conventional scenario because of the presence, in addition to the inflaton, of the Higgs field [8]. During inflation, the curvature perturbation on uniform energy density slices is given, in a spatially flat gauge, by

$$
\zeta=H_{0} \frac{\delta \rho}{\dot{\rho}}=\frac{\dot{\rho}_{\phi}}{\dot{\rho}} \zeta_{\phi}+\frac{\dot{\rho}_{h}}{\dot{\rho}} \zeta_{h},
$$

where we separated the inflaton component $\zeta_{\phi}$ and the Higgs component $\zeta_{h} \equiv H_{0} \delta \rho_{h} / \dot{\rho}_{h}$ [8]. We assume that there is no energy transfer between the Higgs and the inflaton sectors, and that the latter generates, on large scales, the perturbations responsible for the CMB anisotropies and large scale structures. Given these assumptions, 
$\zeta_{\phi}$ and $\zeta_{h}$ are separately conserved on superhorizon scales [33]. As customary, we can decompose $\zeta_{h}$ in Fourier modes. For a given comoving wave number $k$ we have, in a spatially flat gauge,

$$
\begin{aligned}
\zeta_{h}^{(k)} & =H_{0} \frac{\delta \rho_{h}^{(k)}}{\dot{\rho}_{h}}=-\frac{\left[\dot{h}_{\mathrm{cl}} \dot{\delta h_{k}}+V_{\mathrm{eff}}^{\prime}\left(h_{\mathrm{cl}}\right) \delta h_{k}\right]}{3 \dot{h}_{\mathrm{cl}}^{2}} \\
& \left.\simeq \frac{H_{0}^{2}}{\sqrt{2 k^{3}} \dot{h}_{\mathrm{cl}}\left(t_{k}\right)}\right|_{k \ll a H_{0}},
\end{aligned}
$$

where the last analytical approximation is valid for the absolute value of $\zeta_{h}^{(k)}$ in the superhorizon limit, and where we used $\rho_{h}=\dot{h}_{\mathrm{cl}}^{2} / 2+V_{\text {eff }}\left(h_{\mathrm{cl}}\right)$ in the first equality.

The right panel of Fig. 8 compares the numerical result for $\zeta_{h}^{(k)}$ — that is, the first equality in Eq. (52)—with its analytical approximation. The plot, moreover, shows that on superhorizon scales $\zeta_{h}^{(k)}$ stays constant, as it should be since we are working under the underlying assumption that there are no interactions between the Higgs and the inflationary sectors.

\section{Higgs dynamics after inflation}

Assuming instantaneous reheating, the energy density of the inflaton is instantaneously converted into radiation at the end of inflation. The inflaton energy density $\rho_{\mathrm{R}}$ is related to the temperature of the thermal bath by

$$
\rho_{\mathrm{R}}=\frac{\pi^{2} g_{*}}{30} T^{4}
$$

The dynamics after reheating is described by the following system of coupled Higgs-radiation equations:

$$
\begin{aligned}
\ddot{h}_{\mathrm{cl}}+(3 H+\Gamma) \dot{h}_{\mathrm{cl}}+V_{\mathrm{T}}^{\prime}\left(h_{\mathrm{cl}}\right) & =0, \\
\dot{\rho}_{\mathrm{R}}+4 H \rho_{\mathrm{R}} & =\Gamma \rho_{h},
\end{aligned}
$$

where the energy density of the Higgs field is given by

$$
\rho_{h}=\frac{\dot{h}_{\mathrm{cl}}^{2}}{2}+V_{\mathrm{eff}}^{\mathrm{T}}\left(h_{\mathrm{cl}}\right),
$$

and the Hubble parameter is related to the total energy density by

$$
H^{2}=\frac{\rho_{\mathrm{R}}+\rho_{h}}{3 \bar{M}_{\mathrm{Pl}}^{2}} .
$$

The damping factor $\Gamma$ takes into account the Higgs decays at temperature $T$ and represents the energy transportation from the Higgs field to radiation. We use the expression quoted in [8], $\Gamma \simeq 10^{-3} T$. In the following, we shall adopt the assumption of sudden decay approximation. In this approximation-corresponding to $\Gamma=0$ in Eqs. (54) Higgs and radiation evolve separately, and the Higgs decay occurs instantaneously at $H=\Gamma$. The system in Eqs. (54) can be solved using the following boundary conditions. As far as the classical Higgs field is concerned, we use the field values at the end of inflation computed in Sec. III C. The evolution of $\rho_{\mathrm{R}}$, in contrast, starts from the temperature in Eq. (35). ${ }^{9}$ Using the solution for $\rho_{\mathrm{R}}$, Eq. (53) gives the time evolution of the temperature.

After instantaneous reheating, the Higgs potential suddenly changes from the expression in Eq. (32) to the one in Eq. (36), and interactions with the SM bath generate a large thermal mass. If $h_{\text {end }}<h_{\max }^{\mathrm{T}}$, the fall of the Higgs field is rescued by thermal corrections, and its background value starts oscillating around the minimum at zero until the decay becomes efficient at $H=\Gamma$. At this stage, the Higgs component $\zeta_{h}$ of the curvature perturbation is not conserved compared to the value computed in Eq. (52) during inflation. This is because the interactions with the SM plasma that are responsible for the appearance of the thermal mass introduce a nonadiabatic component in the pressure perturbation.

We compute-in a spatially flat gauge, and for a given comoving wave number $k$-the total curvature perturbation after reheating according to

$$
\zeta^{(k)}=H \frac{\delta \rho}{\dot{\rho}}=\frac{\dot{h}_{\mathrm{cl}} \dot{\delta h_{k}}+V_{\mathrm{eff}}^{\mathrm{T}^{\prime}}\left(h_{\mathrm{cl}}\right) \delta h_{k}}{-4 \rho_{\mathrm{R}}-3 \dot{h}_{\mathrm{cl}}^{2}},
$$

where radiation perturbations are set to zero. We compute $\zeta^{(k)}$ numerically at the time of Higgs decay, $H=\Gamma$. After Higgs decay, radiation remains as the only component of the Universe and $\zeta^{(k)}$ is, therefore, conserved.

\section{E. The power spectrum and the PBH abundance}

The curvature power spectrum is given by

$$
\mathcal{P}_{\zeta}=\frac{k^{3}}{2 \pi^{2}}\left|\zeta^{(k)}\right|^{2}
$$

A numerical example is shown in Fig. 9. Its left panel shows that a small $\xi_{H}=-10^{-3}$ has only a minor effect. The cut in the power spectrum at small $k$ arises because of assumption (i): before that classical rolling starts, the Higgs

\footnotetext{
${ }^{9}$ More precisely, conservation of total energy determines the reheating temperature as

$3 \bar{M}_{\mathrm{Pl}}^{2} H_{0}^{2}+\frac{\dot{h}_{\mathrm{cl}}^{2}}{2}+\left.V_{\mathrm{eff}}\left(h_{\mathrm{cl}}\right)\right|_{h_{\mathrm{cl}}=h_{\mathrm{end}}}=\frac{\pi^{2} g_{*}}{30} T^{4}+\frac{\dot{h}_{\mathrm{cl}}^{2}}{2}+\left.V_{\mathrm{eff}}^{\mathrm{T}}\left(h_{\mathrm{cl}}\right)\right|_{h_{\mathrm{cl}}=h_{\mathrm{end}}}$.
}

The approximation in Eq. (35) is valid because the inflaton energy density dominates over the Higgs contribution. 

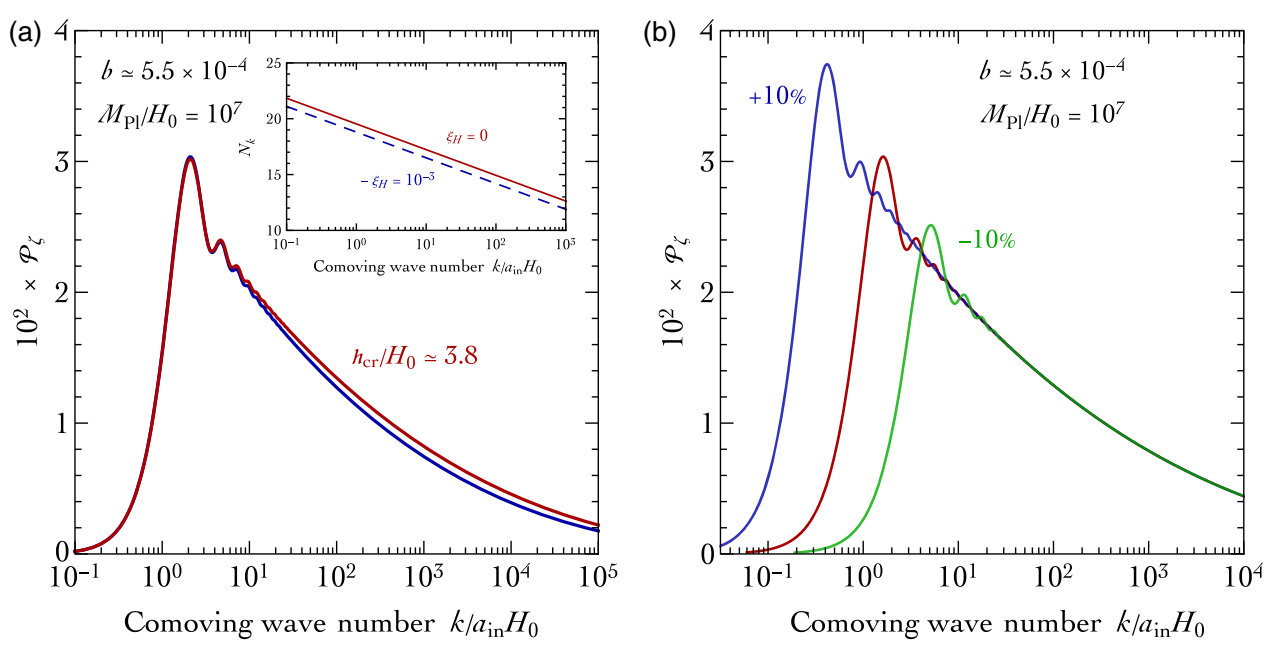

FIG. 9. Left: The power spectrum of Higgs fluctuations produced by rescued Higgs fall for $\xi_{H}=0$ (red curve) and $\xi_{H}=-10^{-3}$ (blue curve), which has a minor impact. In the inset we show the number of $e$-folds at the horizon exit for each mode. Right: How the power spectrum changes when the classicality condition in Eq. (38) is changed by $\pm 10 \%$.

field is away from its minimum and exactly homogeneous. Relaxing this assumption would increase the power spectrum at small $k$.

The right panel shows the significant effect of a small change in the arbitrary order one parameter $c$ that defines the classicality condition in Eq. (38). Reducing $c$ anticipates the initial moment $t_{\text {in }}$ (or equivalently $N_{\text {in }}$ ) where the Higgs starts to roll down the potential. As a consequence, earlier quantum fluctuations get taken into account by our computation of Sec. III C.

Finally, we can now compute the mass and amount of $\mathrm{PBH}$ generated by Higgs fluctuations. The radius of the Hubble horizon or the wavelength of the modes determines the typical mass of the PBHs [34],

$$
M_{\mathrm{PBH}} \approx \frac{\gamma}{2} \frac{M_{\mathrm{Pl}}^{2}}{H_{0}} e^{2 N}
$$

where $N$ is the number of $e$-folds when the $k$ mode leaves the horizon; $\gamma \approx 0.2$ is a correction factor [35]. For example, $H_{0}=10^{12} \mathrm{GeV}$ and $N=20$ gives $M_{\mathrm{PBH}} \approx 10^{-15} M_{\odot}$.

To compute the fraction of the Hubble volume collapsing to PBHs, we need the variance of the smoothed density perturbation over a radius $R, \sigma^{2}(R)=\left\langle\delta^{2}(\boldsymbol{x}, R)\right\rangle$, where $\delta(\boldsymbol{x}, R)=\int \mathrm{d}^{3} x^{\prime} \delta\left(\boldsymbol{x}^{\prime}\right) W\left(\boldsymbol{x}-\boldsymbol{x}^{\prime}, R\right)$ is the density fluctuation smoothed by a window function $W(\boldsymbol{x}, R)$, assumed to be Gaussian,

$$
W(\boldsymbol{x}, R)=\frac{1}{(2 \pi)^{3 / 2} R^{3}} \exp \left(-\frac{|\boldsymbol{x}|^{2}}{2 R^{2}}\right) .
$$

The variance can be computed in terms of density power spectrum $P_{\delta}(k)$, which is related to the curvature perturbation $\zeta$ power spectrum $P_{\zeta}(k)$ as [36]

$$
\begin{aligned}
\sigma^{2}(R) & =\int \mathrm{d} \ln k P_{\delta}(k) \tilde{W}^{2}(k R), \\
& =\int \mathrm{d} \ln k \frac{16}{81}(k R)^{4} P_{\zeta}(k) \tilde{W}^{2}(k R),
\end{aligned}
$$

where $\tilde{W}(k)=\exp \left(-k^{2} / 2\right)$ is the Fourier transform of the window function. Assuming that $\mathrm{PBH}$ are formed when the density perturbation exceeds $\delta_{\text {th }} \approx 0.5$ [37], the fraction of the Universe ending up in PBH is given by the tail of the assumed Gaussian distribution,

$$
\begin{aligned}
& \beta\left(M_{\mathrm{PBH}}\right)=\gamma \int_{\delta_{\mathrm{th}}}^{\infty} \mathrm{d} \delta \frac{1}{\sqrt{2 \pi} \sigma\left(M_{\mathrm{PBH}}\right)} \exp \left(-\frac{\delta^{2}}{2 \sigma^{2}\left(M_{\mathrm{PBH}}\right)}\right) \\
& \stackrel{\sigma \ll \delta_{\text {th }}}{\simeq} \frac{\gamma \sigma}{\sqrt{2 \pi} \delta_{\text {th }}} \exp \left(-\frac{\delta_{\text {th }}^{2}}{2 \sigma^{2}}\right) \text {. }
\end{aligned}
$$

The latter approximation is relevant, given that the obtained power spectra $P_{\zeta}$ are of order $10^{-2}$. We convert $\sigma(R)$ to a function $\sigma\left(M_{\mathrm{PBH}}\right)$, taking into account that the size $R$ is related to the mass $M_{\mathrm{PBH}}$ as $R \approx 2 G M_{\mathrm{PBH}} / \gamma a$. The fraction of $\mathrm{PBH}$ relative to the $\mathrm{DM}$ abundance at given mass, $f_{\mathrm{PBH}}\left(M_{\mathrm{PBH}}\right)$, is given by [36]

$$
f_{\mathrm{PBH}} \approx 2.7 \times 10^{8}\left(\frac{10.75}{g_{*, \text { form }}}\right)^{1 / 4}\left(\frac{\gamma}{0.2} \frac{M_{\odot}}{M_{\mathrm{PBH}}}\right)^{1 / 2} \beta .
$$

The distribution of $\mathrm{PBH}$ as a function of their mass $M_{\mathrm{PBH}}$ is strongly peaked at the value that maximizes $\sigma(M)$, in view of the exponential factor in Eq. (63). In terms of the power spectrum $P_{\zeta}$ this means that the $\mathrm{PBH}$ abundance roughly scales as $e^{-1 / P_{\zeta}}$ and is dominated by the top of the peak of $P_{\zeta}(k)$. The PBH mass distribution is peaked at the value corresponding to the $k$ that maximizes $P_{\zeta}(k)$. The desired abundance is reproduced for $\max P_{\zeta} \sim 10^{-2}$, and slightly 
larger (smaller) values produce way too many (too few) PBH.

This means that, due to the fine-tuned nature of the mechanism (as in all models that can generate $\mathrm{PBH}$ ), the amount and mass of PBH depend in an extremely sensitive way on the uncertain SM and cosmological parameters, mainly the top mass and the Hubble constant $H_{0}$. Furthermore, uncertainties in the computation of black hole formation (such as the value of $\delta_{\text {th }}$ ) imply uncertainties of many orders of magnitude in the PBH density.

For example, a $10 \%$ variation in the order one arbitrary constant $c$ that parametrizes the classicality condition has an order one impact on the power spectrum (Fig. 9), and consequently a huge impact on the $\mathrm{PBH}$ abundance.

In addition to $\mathrm{SM}$ parameters and to $H_{0}$, the $\mathrm{PBH}$ abundance also depends on two extra parameters that characterize the assumed initial condition: constant $h(x)=$ $h_{\text {in }}$ at $N_{\text {in }} e$-folds before the end of inflation, when the approximated classicality condition is satisfied. The next section reconsiders if this assumption is justified.

\section{F. Is a homogeneous Higgs background a sensible assumption?}

The computation was based on assumption (i): at $N_{\text {in }} \approx$ $20 e$-folds before the end of inflation, the Higgs field must be away from its minimum and constant within the presently observable horizon.

\section{Approximate Higgs homogeneity?}

We here show that approximate homogeneity is a natural product of inflation, provided that the SM Higgs potential satisfies certain conditions. Quantum corrections in inflationary (de Sitter) spacetime have been studied in [38], which showed that long wavelength fluctuations can be described by a Fokker-Planck equation for $\rho(h, N)$, the probability of finding the Higgs field at the value $h$ at $N e$-folds of inflation,

$$
-\frac{\partial \rho}{\partial N}=\frac{H_{0}^{2}}{8 \pi^{2}} \frac{\partial^{2} \rho}{\partial h^{2}}+\frac{\partial}{\partial h}\left(\frac{V_{\mathrm{eff}}^{\prime} \rho}{3 H_{0}^{2}}\right) .
$$

The first term on the right-hand side is a diffusion term due to quantum fluctuations while the second term is a drift (or transport) term due to the potential. After some $e$-folds, the distribution converges to its equilibrium value $\rho_{\mathrm{eq}}(h) \propto$ $\exp \left(-8 \pi^{2} V_{\text {eff }}(h) / 3 H^{4}\right)$ [38].

We are interested in the probability of having a roughly constant Higgs away from its minimum $h=0$ within the presently observable horizon. This configuration is a natural outcome of inflationary dynamics provided that the correlation length of Higgs fluctuations is larger than the present horizon. Following [38], the computation of the correlation length is simplified, observing that correlation functions depend on space and time separations respecting the $\mathrm{O}(4,1)$ invariance of de Sitter. Thereby we can compute the evolution of Higgs fluctuations at a fixed point in space and study the correlation as a function of time $t$, or equivalently as a function of the number of $e$-folds $N$. At a large time separation the correlation is well approximated by its dominant exponential and parametrized in terms of a correlation time $\tau_{\text {corr }}$ or equivalently in terms of number of $e$-folds $N_{\text {corr }}=H_{0} \tau_{\text {corr }}$ as [38]

$$
\left\langle h\left(t_{1}, \vec{x}\right) h\left(t_{2}, \vec{x}\right)\right\rangle \simeq\left\langle h^{2}\right\rangle e^{-\left|t_{1}-t_{2}\right| / \tau_{\text {corr }}}=\left\langle h^{2}\right\rangle e^{-\left|N_{1}-N_{2}\right| / N_{\text {corr }}} .
$$

$\mathrm{O}(4,1)$ invariance implies that the spatial correlation length is exponentially large $e^{H N_{\text {corr }}}$, namely that space is exponentially inflated. We demand that $N_{\text {corr }}$ is larger than about 40 in order to produce a smooth region as large as our Universe at $N_{\text {in }} \approx 20 e$-folds before the end of inflation. Before computing numerically $N_{\text {corr }}$ for the SM potential, it is useful to consider some simple limits:

(0) A massless free scalar $h$ is a simple but special case, because it does not "thermalize" to the equilibrium distribution. Rather, it undergoes random walk diffusion. Starting from $h=0$, after $N e$-folds one has $\left\langle h^{2}\right\rangle^{1 / 2}=\sqrt{N} H_{0} / 2 \pi$ and the correlation length in a region with given $\langle h\rangle$ is $N_{\text {corr }}=2 \pi\langle h\rangle / H$. Imposing $N_{\text {corr }}>40$ implies $H_{0}<0.16\langle h\rangle$.

(m) A free scalar with squared mass $m^{2}>0$ fluctuates as $\left\langle h^{2}\right\rangle^{1 / 2}=\sqrt{3 / 2} H^{2} / 2 \pi m$ with correlation length $N_{\text {corr }}=3 H^{2} / m^{2}$ [38]. For $m^{2}=-12 \xi_{H} H^{2}$ one gets $N_{\text {corr }}=-1 / 4 \xi_{H}>40$ for $-0.006<\xi_{H}<0$. Roughly, this will be our final result.

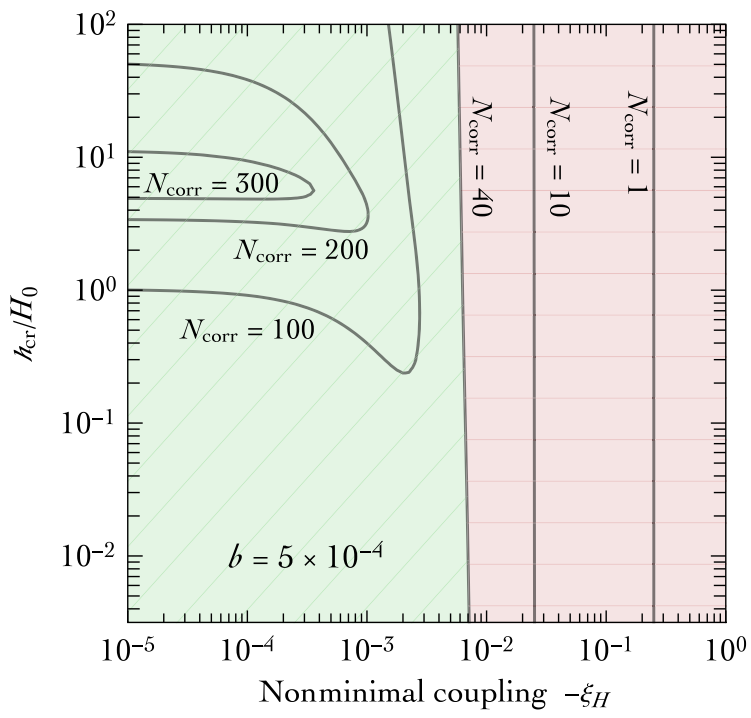

FIG. 10. Contour plot of the correlation length $N_{\text {corr }}$ of Higgs inflationary fluctuations as a function of the main parameters, $\xi_{H}$ and $h_{\text {cr }} / H_{0}$. The needed homogeneous patch, $N_{\text {corr }}>40$, can be obtained in the green region, which corresponds to a small negative $\xi_{H}$. 

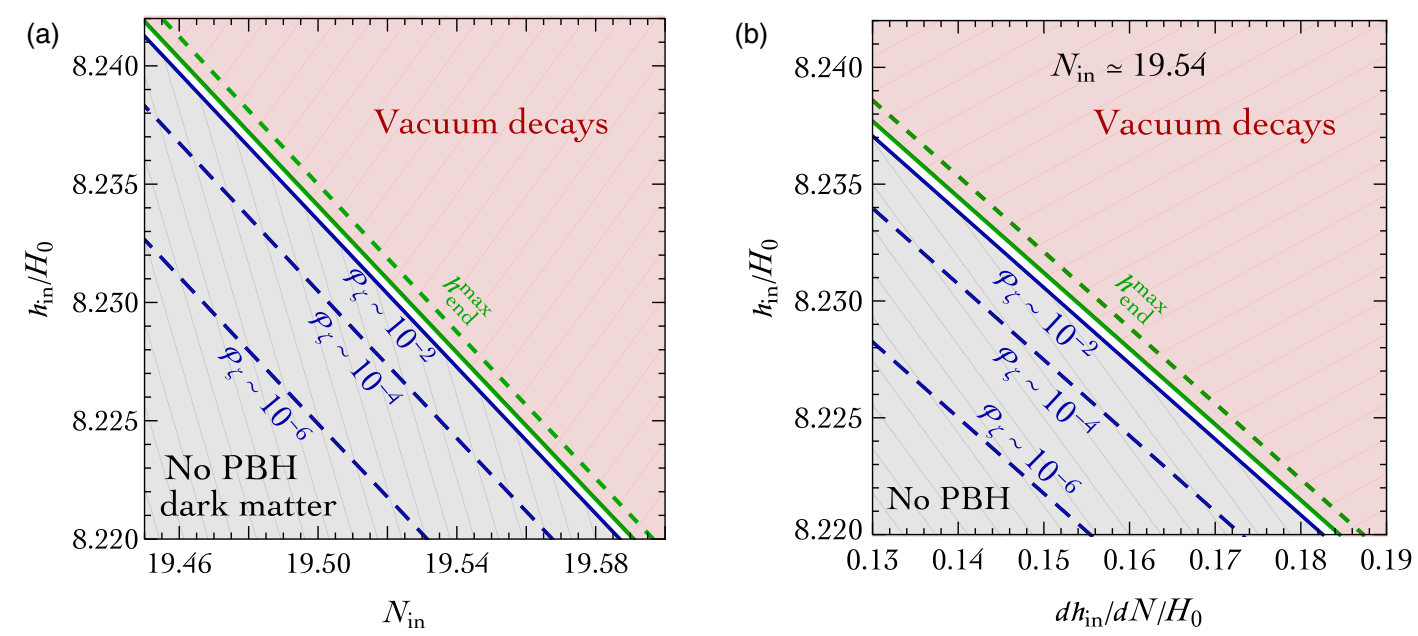

FIG. 11. We fix the SM to $M_{t}=172 \mathrm{GeV}$ [which corresponds to $h_{\mathrm{cr}}=4 \times 10^{12} \mathrm{GeV}, b=0.09 /(4 \pi)^{2}$ ] and $H_{0}=10^{12} \mathrm{GeV}$ and explore the dependence on the parameters that define the assumed initial condition. Left: Final outcome as a function of the homogeneous initial value of the Higgs field $h_{\text {in }}$ at $N_{\text {in }} e$-folds before the end of inflation and of $N_{\text {in }}$. The desired PBH abundance is produced inside the white band. Inflationary fluctuations in $h_{\text {in }}$ are much larger than the needed tuning. Right: We fix $N_{\text {in }}$ and introduce $d h_{\text {in }} / d N$ as an additional parameter.

( $\lambda$ ) A massless scalar with an interaction $\lambda h^{4} / 4$ and $\lambda>0$ fluctuates as $\left\langle h^{2}\right\rangle^{1 / 2}=0.36 H / \lambda^{1 / 4}$ with $N_{\text {corr }}=$ $7.62 / \sqrt{\lambda}$. So $N_{\text {corr }}>40$ for $\lambda<0.036$. This is satisfied in the SM at large energy.

The above two results agree, up to order one factors, approximating a generic $V(h)$ as a massive field with $m^{2}=V^{\prime \prime}$. The precise value of the correlation length can be obtained as proposed in [38]: the dominant exponential that solves Eq. (65) can be computed as the eigenvalue of a Schrödinger-like equation. In the $\mathrm{SM} \lambda_{\text {eff }}(h)$ runs to negative values, making the curvature $V^{\prime \prime}$ negative at large field values; the patch with a large correlation length is created while $h$ is climbing the potential in its region with positive curvature. The result is shown in Fig. 10, and basically agrees with the result anticipated at point $(m): \xi_{H}$ must be negative and small. Such a small $\xi_{H}$ is roughly compatible with the size of quantum corrections to $\xi_{H}$. The running Higgs quartic coupling is small enough that it does not spoil the mechanism, as qualitatively understood at point $(\lambda)$.

In conclusion, a roughly homogeneous Higgs field (up to fluctuation of order $H_{0}$ ) encompassing our whole horizon is a natural outcome of inflation, provided that $\xi_{H}$ is small enough. In a multiverse context, its fine-tuned value that leads to DM as PBH can be justified on an anthropic basis.

\section{Exact Higgs homogeneity?}

Figure 11(a) shows that the initial homogeneous Higgs fields $h(x)=h_{\text {in }}$ at $N=N_{\text {in }} e$-folds before the end of inflation must be tuned to a part in about $10^{-3}$ in order to produce a final $h_{\text {end }}$ close to the maximal value that can be rescued by reheating, as needed to produce a substantial amount of primordial black holes. An interesting PBH abundance is obtained within the narrow strip in Fig. 11(a). Its boundaries have been computed as follows:

(i) A slightly smaller $h_{\text {in }}$ leads to a negligible PBH amount. In Fig. 11(a) we show the peak value of the power spectrum and shade in gray regions where it is below $10^{-2}$.

(ii) A slightly larger $h_{\text {in }}$ leads to a too large $h_{\text {end }}$ not rescued by reheating. In Fig. 11(a) we shade in red regions where $h_{\text {end }}$ is above the maximal value that can be rescued by instantaneous reheating.

In Fig. 11(b) we show that a variation in $\dot{h}_{\text {in }}$ has a smaller effect as it gets redshifted away.

Within the assumption that $h_{\text {in }}$ is homogeneous, its tuned value can be justified on an anthropic basis provided that PBH make all DM $[8,39]$. However, one single fluctuation $\delta h_{\text {in }} \gtrsim 10^{-3} h_{\text {in }}$ away from the assumed perfect homogeneity can lead to one vacuum decay bubble that, after inflation, expands engulfing the observable Universe.

As discussed previously, inflation can produce an approximate homogeneity within a large patch, but up to fluctuations of order $\delta h_{\text {in }} \sim H_{0} / 2 \pi{ }^{10}$ Additional fluctuations in $\dot{h}_{\text {in }}$ are less significant and we ignore them. The Hubble constant $H_{0}$ cannot be significantly reduced, for two reasons: First, $H_{0} \gtrsim h_{\mathrm{cr}}$ is needed to keep the Higgs fluctuating around the top of its potential barrier, until it starts to classically roll down. Second, it would suppress the unwanted prefall fluctuations $\delta h_{\text {in }}$ at the price of

\footnotetext{
${ }^{10}$ Technically, such fluctuations appear in our equations when (motivated, e.g., by the arbitrariness in the classicality condition) we start from a different initial time: this shifts the position and the height of the presumed peak of the power spectrum, and thereby the abundance and mass of PBHs.
} 
suppressing postfall fluctuations that generate $\mathrm{PBHs}$; see Eq. (52).

Thereby we must take into account the effect of fluctuations in the initial $h(x)$. Pictorially, the status of the Universe should now be represented in Fig. 10 not by a point (which can lie in the desired region), but by a dot, much thicker than the desired region.

This is a problem because, at $N_{\text {in }} \approx 20 e$-folds before the end of inflation (which needs at least $60 e$-folds), the present Universe is composed by about $e^{120}$ causally disconnected regions. Within each region, the probability that the Higgs fluctuates to the desired tuned $h_{\text {in }}$ is about $10^{-2-3}$.

Underfluctuations produce a negligible $\mathrm{PBH}$ abundance. On small scales this is not a problem: only the average matters. Accretion of black holes after their formation would increase the average. On large scales of the order of the present horizon (those probed by observations that find a DM density more homogeneous than what could be justified anthropically), fluctuations of $h(x)$ would produce a PBH density that is not homogeneous.

The effect of overfluctuations is much worse. As in a cosmic Russian roulette, a too large $h_{\text {in }}$ in one of the $e^{120}$ regions can form a vacuum decay bubble that, after the end of inflation, engulfs the whole Universe (a general relativity computation finds that innocuous bubbles that shrink and/ or expand behind a black hole horizon can form, but together with dangerous ones [8]). The probability of avoiding vacuum decay is roughly estimated as $\wp \sim 2^{-e^{120}}$.

As we now discuss, this unlikely possibility cannot be justified on an anthropic basis.

To explain why, let us start from an analogous anthropic argument considered by Weinberg [40]: if observers exist only where the cosmological constant $\Lambda$ is small enough to allow their development (at the price of a tuning with probability $\wp_{\Lambda} \sim 10^{-120}$, which is possible in multiverse with more than $10^{120}$ different vacua), they should expect to see a cosmological constant around the anthropic bound. Once the desired Universe with small $\Lambda$ is formed, it is relatively safe. Technically, the timescale for the variation of the $\wp_{\Lambda}$ is the Hubble scale, presently $10^{10} \mathrm{yr}$.

Within the "rescued Higgs fall" mechanism, the probability $\wp$ to form DM but no vacuum decay is much smaller. This might be by itself a problem, unless one relies on eternal inflation and argues that $1 / \wp$ is smaller than infinity. The same Weinberg-like argument leads to expectations incompatible with experience. Indeed, the timescale for the growth of $1 / \wp$ is very short. An observer that justifies its lucky survival to vacuum decay as needed for its existence should expect to be immediately executed by an expanding vacuum decay bubble, given that it arises with statistical certainty in the extra regions which continuously enter in causal contact with the observer due to the Universe expansion.

Needless to say, multiverse probability is a shaky concept, plagued by infinities. Nevertheless, the problem seems worrying enough that one wonders if it can be avoided or alleviated.

One possibility is devising a mechanism that suppresses vacuum decay by rescuing the Higgs more efficiently than the thermal barrier considered in $[8,10]$. For example, a nonthermal distribution ${ }^{11}$ or an inflation that initially decays to the SM particles more coupled to $h$ increases its thermal mass. The green dashed line in Fig. 11 shows the extra rescued region imposing what we believe is the most optimistic possibility: $h_{\text {end }}<h_{\text {end }}^{\max }$ with $V_{\text {eff }}\left(h_{\text {end }}^{\max }\right)=0$. Namely, we demand that the negative Higgs potential energy remains smaller than the inflaton potential $V_{0}=3 \bar{M}_{\mathrm{Pl}}^{2} H_{0}^{2}$; otherwise, nothing can stop the Higgs fall. Figure 11 shows that a more efficient rescue mechanism would not qualitatively change the picture. Alternatively, some mechanism beyond the SM could prepare the Higgs in the homogeneous state needed for the rescued Higgs fall mechanism. A study of this possibility goes beyond the scope declared in the title of this paper, where we wanted to see if some mechanism can generate dark matter within the Standard Model.

\section{CONCLUSIONS}

We critically reexamined two different dark matter candidates that do not require new physics beyond the Standard Model.

The first is a hexaquark $\mathcal{S}=$ uuddss dibaryon, which (being a spin 0 isospin singlet) might have a QCD binding energy large enough to make it lighter than $M_{\mathcal{S}}<$ $1.876 \mathrm{GeV}$ and thereby stable because all possible decay modes are kinematically closed. In Sec. II A we estimated its mass at the light of recently measured tetraquarks. We found that $\mathcal{S}$ could be light enough, possibly as light as $1.2 \mathrm{GeV}$, although we cannot provide a precise mass. In Sec. II B we computed its cosmological relic density, finding that it can reproduce the desired DM density if $M_{\mathcal{S}} \approx 1.2 \mathrm{GeV}$, while larger masses lead to smaller abundances. The dominant process that keeps $\mathcal{S}$ in thermal equilibrium is the scattering of two strange baryons, whose abundance gets Boltzmann suppressed at temperatures smaller than the strange quark mass, leading to $\mathcal{S}$ decoupling at the temperature $T_{\mathrm{dec}} \approx 25 \mathrm{MeV}$ where $\mathcal{S}$ has the desired abundance. However, in Sec. II C we find, following the strategy of [2], that such a light $\mathcal{S}$ is excluded, because nucleons inside nuclei would bind in $\mathcal{S}$ faster than what is allowed by Super-Kamiokande bounds on the stability of oxygen. We reached this conclusion at the light of recent global fits of nuclear potentials used to compute the nuclear wave function of oxygen, which indicate that nucleons are close enough to make $\mathcal{S}$ production too fast. Both $\mathcal{S}$ and nuclei can be stable for $M_{\mathcal{S}} \approx 1.87 \mathrm{GeV}$; however, this mass leads to a relic $\mathcal{S}$ abundance much

\footnotetext{
${ }^{11}$ We thank the authors of [8] for this suggestion.
} 
smaller than the DM abundance. Among sparse comments, we mention that direct detection of $\mathcal{S}$ on an antimatter target gives an annihilation signal.

In the second part of the paper, we considered the proposal of [8]: given that the SM Higgs potential can be unstable at large field values, during inflation the Higgs might fall from an assumed homogeneous vacuum expectation value $h$ beyond the potential barrier toward the Planck scale. If the fall is tuned such that $h$ almost reaches the maximal value that can be rescued by thermal effects, this process generates small-scale inhomogeneities that form primordial black holes. While DM as PBH seems excluded in the proposed mass range (just above the bound on Hawking radiation), the proposal is interesting enough to deserve further scrutiny. We confirmed the computations of [8] and extended them, adding a nonminimal coupling $\xi_{H}$ of the Higgs to gravity. We find that inflationary fluctuations can generate a quasihomogenous $h$ only if $\xi_{H}$ is as small as allowed by quantum corrections that unavoidably generate it. Furthermore, we find in Sec. III F that the amount and mass of PBH depend on an extremely sensitive way on the uncertain SM and cosmological parameters, including two extra parameters introduced as assumptions: the Higgs starts falling from a homogeneous value at a given moment during inflation. The assumed homogeneity is, however, not the typical state present during inflation, where the Higgs has fluctuations of order Hubble. This is important for the present mechanism because it has a Russian roulette feature: the Universe is eaten by a vacuum decay bubble if the Higgs fluctuates to a value too high to be rescued by thermal effects in one of the $e^{120}$ causally disconnected patches in which the present horizon is divided while the Higgs starts falling. The probability of obtaining the observed Universe is so small, about
$(1 / 2)^{e^{120}}$, that trying to justify it through anthropic considerations leads to the issues discussed in Sec. III F.

We conclude that tentative searches and interpretations of dark matter as a phenomenon beyond the Standard Model remains a justified field.

\section{ACKNOWLEDGMENTS}

We thank G. Ballesteros, I. Bombaci, A. Bonaccorso, A. Francis, A. Gnech, J. Green, M. Karliner, D. Racco, and Y. Wang for discussions and M. Redi for participating in an early stage of this project. We especially thank G. Farrar for discussions about $\mathrm{S}$ breakup cross sections and A. Riotto for many useful suggestions at all stages of this work, and in particular for clarifications about nonadiabaticity of $\zeta_{h}$ because of interactions with the plasma. A. U. thanks all participants in the Dark Matter Coffee Break at SISSA for many stimulating discussions about the physics of primordial black holes. This work was supported by the ERC Grant No. 669668 NEO-NAT.

Note added.-G. Farrar in arXiv: 1805.03723 proposed that a co-stable $\mathcal{S}$ dark matter with $1.86 \mathrm{GeV}<M_{\mathcal{S}}<1.88 \mathrm{GeV}$ could be produced with roughly the correct relic abundance at the QCD phase transition at $T \approx 150 \mathrm{MeV}$. We have not considered this possibility because this abundance would be washed out by thermal equilibrium through the $\mathcal{S}$ breakup reactions in Eq. (18). To avoid this conclusion, the $\mathcal{S}$ breaking cross sections should be $\sim 10$ orders of magnitude smaller than the naive QCD estimate given by the $\mathcal{S}$ radius squared. We view this as a too extreme possibility given that - while some special suppression could arise at low energy assuming appropriate nucleon potentials - one would need such a strong suppression at $T \approx 150 \mathrm{MeV}$, where the simpler QCD physics is relevant.
[1] R. L. Jaffe, Strangelets, Nucl. Phys. B, Proc. Suppl. 24B, 8 (1991).

[2] G. R. Farrar and G. Zaharijas, Nuclear and nucleon transitions of the H dibaryon, Phys. Rev. D 70, 014008 (2004).

[3] G. R. Farrar, Stable sexaquark, arXiv:1708.08951.

[4] G. R. Farrar, 6-quark dark matter, Proc. Sci., ICRC2017 (2018) 929 [arXiv:1711.10971].

[5] D. Lonardoni, A. Lovato, S. C. Pieper, and R. B. Wiringa, Variational calculation of the ground state of closed-shell nuclei up to $A=40$, Phys. Rev. C 96, 024326 (2017).

[6] A. Katz, J. Kopp, S. Sibiryakov, and W. Xue, Femtolensing by dark matter revisited, arXiv:1807.11495.

[7] H. Niikura, M. Takada, N. Yasuda, R. H. Lupton, T. Sumi, S. More, A. More, M. Oguri, and M. Chiba, Microlensing constraints on primordial black holes with the Subaru/HSC Andromeda observation, arXiv:1701.02151.
[8] J. R. Espinosa, D. Racco, and A. Riotto, A Cosmological Signature of the Standard Model Higgs Vacuum Instability: Primordial Black Holes as Dark Matter, Phys. Rev. Lett. 120, 121301 (2018).

[9] G. Degrassi, S. Di Vita, J. Elias-Miro, J. R. Espinosa, G. F. Giudice, G. Isidori, and A. Strumia, Higgs mass and vacuum stability in the Standard Model at NNLO, J. High Energy Phys. 08 (2012) 098; D. Buttazzo, G. Degrassi, P. P. Giardino, G. F. Giudice, F. Sala, A. Salvio, and A. Strumia, Investigating the nearcriticality of the Higgs boson, J. High Energy Phys. 12 (2013) 089.

[10] J. R. Espinosa, G. F. Giudice, E. Morgante, A. Riotto, L. Senatore, A. Strumia, and N. Tetradis, The cosmological Higgstory of the vacuum instability, J. High Energy Phys. 09 (2015) 174. 
[11] D. Z. Freedman, I. J. Muzinich, and E. J. Weinberg, On the energy-momentum tensor in gauge field theories, Ann. Phys. (N.Y.) 87, 95 (1974).

[12] A. Esposito, A. Pilloni, and A. D. Polosa, Multiquark resonances, Phys. Rep. 668, 1 (2017).

[13] A. De Rujula, H. Georgi, and S. Glashow, Hadron masses in a gauge theory, Phys. Rev. D 12, 147 (1975).

[14] L. Maiani, F. Piccinini, A. D. Polosa, and V. Riquer, A New Look at Scalar Mesons, Phys. Rev. Lett. 93, 212002 (2004).

[15] S. R. Beane, E. Chang, W. Detmold, H. W. Lin, T. C. Luu, K. Orginos, A. Parreno, M. J. Savage, A. Torok, and A. Walker-Loud, The deuteron and exotic two-body bound states from lattice QCD, Phys. Rev. D 85, 054511 (2012).

[16] S. R. Beane, E. Chang, S. D. Cohen, W. Detmold, H. W. Lin, T. C. Luu, K. Orginos, A. Parreno, M. J. Savage, and A. Walker-Loud, Light nuclei and hypernuclei from quantum chromodynamics in the limit of SU(3) flavor symmetry, Phys. Rev. D 87, 034506 (2013).

[17] A. Francis, J. R. Green, P. M. Junnarkar, C. Miao, T. D. Rae, and H. Wittig, Lattice QCD study of the H dibaryon using hexaquark and two-baryon interpolators, arXiv:1805.03966.

[18] P. E. Shanahan, A. W. Thomas, and R. D. Young, Mass of the H-Dibaryon, Phys. Rev. Lett. 107, 092004 (2011).

[19] A. Selem and F. Wilczek, Hadron systematics and emergent diquarks, in New Trends in HERA Physics 2005: Proceedings of the Ringberg Workshop (World Scientific, Singapore, 2006).

[20] Planck Collaboration, Planck 2015 results. XIII. Cosmological parameters, Astron. Astrophys. 594, A13 (2016).

[21] D. Hooper and S. D. McDermott, Robust constraints and novel gamma-ray signatures of dark matter that interacts strongly with nucleons, Phys. Rev. D 97, 115006 (2018).

[22] T. Emken and C. Kouvaris, How blind are underground and surface detectors to strongly interacting Dark Matter?, Phys. Rev. D 97, 115047 (2018).

[23] R. Barkana, Possible interaction between baryons and darkmatter particles revealed by the first stars, Nature (London) 555, 71 (2018).

[24] R. H. Cyburt, B. D. Fields, V. Pavlidou, and B. D. Wandelt, Constraining strong baryon dark matter interactions with primordial nucleosynthesis and cosmic rays, Phys. Rev. D 65, 123503 (2002).

[25] Super-Kamiokande Collaboration, Search for dinucleon decay into pions at Super-Kamiokande, Phys. Rev. D 91, 072009 (2015).

[26] Super-Kamiokande Collaboration, Search for Nucleon and Dinucleon Decays with an Invisible Particle and a Charged
Lepton in the Final State at the Super-Kamiokande Experiment, Phys. Rev. Lett. 115, 121803 (2015).

[27] Super-Kamiokande Collaboration, Measurement of a small atmospheric muon-neutrino/electron-neutrino ratio, Phys. Lett. B 433, 9 (1998).

[28] N. Isgur and G. Karl, Positive parity excited baryons in a quark model with hyperfine interactions, Phys. Rev. D 19, 2653 (1979).

[29] O. Hen, G. A. Miller, E. Piasetzky, and L. B. Weinstein, Nucleon-nucleon correlations, short-lived excitations, and the quarks within, Rev. Mod. Phys. 89, 045002 (2017).

[30] ATLAS, CMS, and TeVatron Collaborations, First combination of Tevatron and LHC measurements of the top-quark mass, arXiv:1403.4427; ATLAS conference note ATLASCONF-2017-071; S. Menke (on behalf of CMS and ATLAS Collaborations), in Proceedings of Moriond 2018, https:// indico.in2p3.fr/event/16579/contributions/60815/ attachments/47187/59266/moriondew2018.pdf.

[31] J. M. Bardeen, P. J. Steinhardt, and M. S. Turner, Spontaneous creation of almost scale-free density perturbations in an inflationary universe, Phys. Rev. D 28, 679 (1983).

[32] D. Wands, K. A. Malik, D. H. Lyth, and A. R. Liddle, New approach to the evolution of cosmological perturbations on large scales, Phys. Rev. D 62, 043527 (2000).

[33] K. A. Malik and D. Wands, Adiabatic and entropy perturbations with interacting fluids and fields, J. Cosmol. Astropart. Phys. 02 (2005) 007.

[34] K. Jedamzik and J. C. Niemeyer, Primordial black hole formation during first order phase transitions, Phys. Rev. D 59, 124014 (1999).

[35] B.J. Carr, The primordial black hole mass spectrum, Astrophys. J. 201, 1 (1975).

[36] M. Sasaki, T. Suyama, T. Tanaka, and S. Yokoyama, Primordial black holes: Perspectives in gravitational wave astronomy, Classical Quantum Gravity 35, 063001 (2018).

[37] I. Musco, J. C. Miller, and L. Rezzolla, Computations of primordial black hole formation, Classical Quantum Gravity 22, 1405 (2005).

[38] A. A. Starobinsky and J. Yokoyama, Equilibrium state of a self-interacting scalar field in the de Sitter background, Phys. Rev. D 50, 6357 (1994).

[39] See, e.g., M. Tegmark, A. Aguirre, M. Rees, and F. Wilczek, Dimensionless constants, cosmology and other dark matters, Phys. Rev. D 73, 023505 (2006).

[40] S. Weinberg, Anthropic Bound on the Cosmological Constant, Phys. Rev. Lett. 59, 2607 (1987). 